Pacific

Journal of

Mathematics

STILL ANOTHER APPROACH TO THE BRAID ORDERING

PATRICK DEHORNOY 


\title{
STILL ANOTHER APPROACH TO THE BRAID ORDERING
}

\author{
PATRICK DEHORNOY
}

\begin{abstract}
We develop a new approach to the linear ordering of the braid group $B_{n}$, based on investigating its restriction to the $\operatorname{set} \operatorname{Div}\left(\Delta_{n}^{d}\right)$ of all divisors of $\Delta_{n}^{d}$ in the monoid $B_{\infty}^{+}$, that is, to positive $\boldsymbol{n}$-braids whose normal form has length at most $d$. In the general case, we compute several numerical parameters attached with the finite orders $\operatorname{Div}\left(\Delta_{n}^{d}\right)$. In the case of 3 strands, we moreover give a complete description of the increasing enumeration of $\operatorname{Div}\left(\Delta_{3}^{d}\right)$. We deduce a new and especially direct construction of the ordering on $B_{3}$, and a new proof of the result that its restriction to $B_{3}^{+}$is a well-ordering of ordinal type $\omega^{\omega}$.
\end{abstract}

This paper investigates the connection between the Garside structure of Artin's braid groups and their distinguished linear ordering, sometimes called the Dehornoy ordering. This leads to a new, alternative construction of the ordering.

Artin's braid groups $B_{n}$ are endowed with several interesting combinatorial structures. One of them stems from Garside's analysis [1969] and is now known as a Garside structure [Dehornoy 2002; McCammond 2005]. It describes $B_{n}$ as the group of fractions of a monoid $B_{n}^{+}$with a rich divisibility theory. This theory gives a unique normal decomposition of every braid in $B_{n}$ into simple braids, which are the divisors of Garside's fundamental braid $\Delta_{n}$, a finite family of $B_{n}^{+}$that is in oneto-one correspondence with the permutations of $n$ objects. One obtains a natural graduation of the monoid $B_{n}^{+}$by considering the family $\operatorname{Div}\left(\Delta_{n}^{d}\right)$ of all divisors of $\Delta_{n}^{d}$, which also are the elements of $B_{n}^{+}$whose normal forms have length at most $d$.

On the other hand, the braid groups are equipped with a distinguished linear ordering which is compatible with multiplication on the left and admits a simple combinatorial characterization [Dehornoy 1994]: a braid $x$ is smaller than another braid $y$ if, among all expressions of the quotient $x^{-1} y$ in the standard generators $\sigma_{i}$, there exists at least one expression in which the generator $\sigma_{m}$ with maximal (or minimal) index $m$ appears only positively, that is, $\sigma_{m}$ occurs, but $\sigma_{m}^{-1}$ does not. Several deep results about that ordering have been proved, for example, that

MSC2000: primary 20F36; secondary 05A05, 20F60.

Keywords: braid group, orderable group, well-ordering, normal form, fundamental braid. 
its restriction to $B_{\infty}^{+}$is a well-ordering. A number of equivalent constructions are known [Dehornoy et al. 2002].

Although both are combinatorial, the previous structures remain mostly unconnected - and connecting them is among the most natural questions of braid combinatorics. For degree 1, that is, for simple braids, the linear ordering corresponds to a lexicographical ordering of the associated permutations [Dehornoy 1999]. But this connection does not extend to higher degrees, and almost nothing is known about the restriction of the linear ordering to positive braids of a given degree. In particular, no connection is known between the Garside normal form and the alternative normal form constructed by S. Burckel [1997; 1999; 2001] which makes comparison with respect to the linear ordering easy. For example, the Garside normal form of $\Delta_{3}^{2 d}$ is $\left(\sigma_{1} \sigma_{2} \sigma_{1}\right)^{2 d}$, while its Burckel normal form is $\left(\sigma_{2} \sigma_{1}^{2} \sigma_{2}\right)^{d} \sigma_{1}^{2 d}$.

This paper investigates the finite linearly ordered sets $\left(\operatorname{Div}\left(\Delta_{n}^{d}\right),<\right)$. A nice way of thinking about this structure is to view the increasing enumeration of $\operatorname{Div}\left(\Delta_{n}^{d}\right)$ as a distinguished path from 1 to $\Delta_{n}^{d}$ in the Cayley graph of $B_{n}$. Completely describing this path would arguably solve optimally the rather vague task of connecting the Garside and the ordered structures of braid groups. The combinatorics of such a description seems to be extremely intricate, and it remains out of reach for the moment, but we prove partial results in this direction.

(i) In the general case, we determine some numerical parameters associated with $\left(\operatorname{Div}\left(\Delta_{n}^{d}\right),<\right)$, which in some sense measure its size. For small values of $n$ and $d$, we find explicit values.

(ii) In the special case $n=3$, we completely describe the increasing enumeration of $\left(\operatorname{Div}\left(\Delta_{n}^{d}\right),<\right)$.

Specifically, the parameters we investigate are the complexity and the heights. The complexity $c\left(\Delta_{n}^{d}\right)$ is defined as the maximal number of $\sigma_{n-1}$ occurring in an expression of $\Delta_{n}^{d}$ containing no $\sigma_{n-1}^{-1}$. We connected the complexity with the termination of the handle reduction algorithm in [Dehornoy 1997], but left its determination as an open question. The $r$-height $h_{r}\left(\Delta_{n}^{d}\right)$ is defined to be the number of $r$-jumps in the increasing enumeration of $\left(\operatorname{Div}\left(\Delta_{n}^{d}\right),<\right.$ ) (augmented by 1 ), where the term $r$-jump refers to some natural filtration of the linear ordering $<$ by a sequence of partial orderings $<_{r}$. When $r$ increases, the $r$-jumps are higher and higher, so $h_{r}\left(\Delta_{n}^{d}\right)$ counts how many big jumps exist in $\left(\operatorname{Div}\left(\Delta_{n}^{d}\right),<\right)$. Here, we prove that the complexity $c\left(\Delta_{n}^{d}\right)$ equals the height $h_{n-1}\left(\Delta_{n}^{d}\right)$ (Proposition 2.19), and that, for each $r$, the $r$-height $h_{r}\left(\Delta_{n}^{d}\right)$ is the number of divisors of $\Delta_{n}^{d}$ whose $d$-th factor of the normal form is right divisible by $\Delta_{r}$ (Proposition 3.11). Together with the combinatorial results of [Dehornoy 2007], this allows for computing the explicit values listed in Table 1, and for establishing various inductive formulas (Propositions 3.15 and 3.17, among others). 
Besides the enumerative results, we also prove a general structural result that connects the ordered set $\left(\operatorname{Div}\left(\Delta_{n}^{d}\right),<\right)$ with subsets of $\left(\operatorname{Div}\left(\Delta_{n-1}^{d}\right),<\right)($ Corollary 3.6). This result suggests an inductive method for directly constructing the increasing enumeration of $\left(\operatorname{Div}\left(\Delta_{n}^{d}\right),<\right)$ starting from those of $\left(\operatorname{Div}\left(\Delta_{n-1}^{d}\right),<\right)$ and $\left(\operatorname{Div}\left(\Delta_{n}^{d-1}\right),<\right)$. This approach is completed here for $n=3$ (Proposition 4.6). In some sense, 3 strand braids are simple objects, and the result may appear as of only modest interest; however, the order on $B_{3}^{+}$is a well-ordering of ordinal type $\omega^{\omega}$ and hence not such a simple object. The interesting point is that this approach leads to a new, alternative construction of the braid ordering, with, in particular, a new and simple proof for the so-called Comparison Property at the heart of the construction (it guarantees the ordering's linearity). In this way, one obtains not just another ordering construction among many [Dehornoy et al. 2002] but, arguably, the optimal one. After the initial inductive definition is correctly stated, it makes all proofs simple and also makes explicit the connection to the Garside structure.

The paper is organized as follows. After an introductory section recalling basic properties and setting the notation, we introduce the parameters $c\left(\Delta_{n}^{d}\right)$ and $h_{r}\left(\Delta_{n}^{d}\right)$ in Section 2 and establish how they are connected. In Section 3, we connect in turn $h_{r}\left(\Delta_{n}^{d}\right)$ to the number of $n$-braids whose $d$-th factor in the normal form satisfies certain constraints, and deduce explicit values. Finally, in Section 4, we study $\left(\operatorname{Div}\left(\Delta_{3}^{d}\right),<\right)$, describe its increasing enumeration, and construct its braid ordering.

\section{Background and preliminary results}

Our notation is standard, and we refer to textbooks like [Birman 1974] or [Epstein et al. 1992] for basic results about braid groups. We recall that the $n$ strand braid group $B_{n}$ is defined for $n>1$ by the presentation

$$
B_{n}=\left\langle\begin{array}{ccc}
\sigma_{i} \sigma_{j}=\sigma_{j} \sigma_{i} & \text { for }|i-j| \geqslant 2 \\
\sigma_{1}, \ldots, \sigma_{n-1} ; & \sigma_{i} \sigma_{j} \sigma_{i}=\sigma_{j} \sigma_{i} \sigma_{j} & \text { for }|i-j|=1
\end{array}\right\rangle,
$$

while, for $n=1$, we let $B_{1}$ be the trivial group. The next group $B_{2}$ is freely generated by $\sigma_{1}$. The elements of $B_{n}$ are called $n$ strand braids, or simply $n$-braids. We use $B_{\infty}$ for the group generated by an infinite sequence of $\sigma_{i}$ 's subject to the relations of (1-1), that is, the direct limit of all $B_{n}$ 's with respect to the inclusion of $B_{n}$ into $B_{n+1}$.

By definition, every $n$-braid $x$ admits (infinitely many) expressions in terms of the generators $\sigma_{i}, 1 \leqslant i<n$. Such an expression is called an $n$ strand braid word. Two braid words $w, w^{\prime}$ representing the same braid are said to be equivalent; the braid represented by a braid word $w$ is denoted $[w]$.

1A. Positive braids and the element $\Delta_{n}$. We denote by $B_{n}^{+}$the monoid admitting the presentation (1-1), and by $B_{\infty}^{+}$the union (direct limit) of all $B_{n}^{+}$'s. The elements 
of $B_{n}^{+}$are called positive $n$-braids. In $B_{\infty}^{+}$, no element except 1 is invertible, and we have a natural notion of divisibility:

Definition 1.1. For $x, y$ in $B_{n}^{+}$, we say that $x$ is a left divisor of $y$, denoted $x \preccurlyeq y$, or, equivalently, that $y$ is a right multiple of $x$, if $y=x z$ holds for some $z$ in $B_{n}^{+}$. We denote by $\operatorname{Div}(y)$ the (finite) set of all left divisors of $y$ in $B_{n}^{+}$.

The monoid $B_{n}^{+}$is not commutative for $n \geqslant 3$, and therefore there are distinct, but symmetric, notions of a right divisor and a left multiple; however, we shall mostly use left divisors. Note that $x$ is a (left) divisor of $y$ in the sense of $B_{n}^{+}$if and only if it is a (left) divisor in the sense of $B_{\infty}^{+}$, so there is no need to specify the index $n$.

According to Garside theory [1969], $B_{n}^{+}$equipped with the left divisibility relation is a lattice: any two positive $n$-braids $x, y$ admit a greatest common left divisor $\operatorname{gcd}(x, y)$, and a least common right multiple $\operatorname{lcm}(x, y)$. A special role is played by the lcm $\Delta_{n}$ of $\sigma_{1}, \ldots, \sigma_{n-1}$, which can be defined inductively by

$$
\Delta_{1}=1, \quad \Delta_{n}=\sigma_{1} \sigma_{2} \ldots \sigma_{n-1} \Delta_{n-1} .
$$

It is well known that $\Delta_{n}^{2}$ belongs to the center of $B_{n}$ (and even generates it for $n \geqslant 3$ ), and that the flip automorphism $\phi_{n}$ of $B_{n}$ corresponding to conjugation by $\Delta_{n}$ exchanges $\sigma_{i}$ and $\sigma_{n-i}$ for $1 \leqslant i \leqslant n-1$.

In $B_{n}^{+}$, the left and the right divisors of $\Delta_{n}$ coincide, and they make a finite sublattice of $\left(B_{n}^{+}, \preccurlyeq\right)$ with $n$ ! elements. These braids will be called simple. When braid words are represented by diagrams as mentioned in Figure 1, simple braids are those positive braids that can be represented by a diagram in which any two strands cross at most once.

By mapping $\sigma_{i}$ to the transposition $(i, i+1)$, one defines a surjective homomorphism $\pi$ of $B_{n}$ onto the symmetric group $\mathfrak{S}_{n}$. The restriction of $\pi$ to simple braids is a bijection: for every permutation $f$ of $\{1, \ldots, n\}$, there exists exactly one

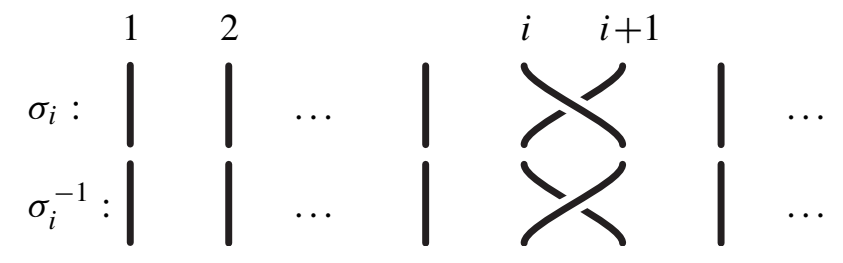

Figure 1. One associates to every $n$ strand braid word $w$ an $n$ strand braid diagram by stacking elementary diagrams as above. Two braid words are equivalent if and only if the associated diagrams are the projections of ambient isotopic figures in $\mathbb{R}^{3}$, that is, one can deform one diagram into the other without allowing the strands to cross or moving the endpoints. 
simple braid $x$ satisfying $\pi(x)=f$. It follows that the number of simple $n$-braids is $n$ !.

Example 1.2. The set $\operatorname{Div}\left(\Delta_{3}\right)$ consists of six elements, namely $1, \sigma_{1}, \sigma_{2}, \sigma_{2} \sigma_{1}$, $\sigma_{1} \sigma_{2}$, and $\Delta_{3}$. In examples, we shall often use the shorter notation a for $\sigma_{1}, \mathrm{~b}$ for $\sigma_{2}$, etc. Thus, the six simple 3-braids are $1, \mathrm{a}, \mathrm{b}, \mathrm{ba}, \mathrm{ab}, \mathrm{aba}$.

1B. The normal form. For each positive $n$-braid $x$ distinct from 1 , the simple braid $\operatorname{gcd}\left(x, \Delta_{n}\right)$ is the maximal simple left divisor of $x$, and we obtain a distinguished expression $x=x_{1} x^{\prime}$ with $x_{1}$ simple. By decomposing $x^{\prime}$ in the same way and iterating, we obtain the so-called normal expression [El-Rifai and Morton 1994; Epstein et al. 1992].

Definition 1.3. A sequence $\left(x_{1}, \ldots, x_{d}\right)$ of simple $n$-braids is said to be normal if, for each $k$, one has $x_{k}=\operatorname{gcd}\left(\Delta_{n}, x_{k} \ldots x_{d}\right)$.

Clearly, each positive braid admits a unique normal expression. It will be convenient to consider the normal expression as unbounded on the right by completing it with as many trivial factors 1 as needed. In this way, we can speak of the $d$-th factor (in the normal form) of $x$ for each positive braid $x$. We say that a positive braid has degree $d$ if $d$ is the largest integer such that the $d$-th factor of $x$ is not 1 . We shall use the following two properties of the normal form:

Lemma 1.4 [El-Rifai and Morton 1994]. Suppose $\left(x_{1}, \ldots, x_{d}\right)$ is sequence of simple $n$-braids. It is normal if and only if, for each $k<d$, each $\sigma_{i}$ that divides $x_{k+1}$ on the left divides $x_{k}$ on the right.

Lemma 1.5 [El-Rifai and Morton 1994]. For x a positive braid in $B_{n}^{+}$, the following are equivalent:

(i) The braid $x$ belongs to $\operatorname{Div}\left(\Delta_{n}^{d}\right)$, that is, is a (left or right) divisor of $\Delta_{n}^{d}$;

(ii) The degree of $x$ is at most $d$.

Example 1.6. There are 19 divisors of $\Delta_{3}^{2}$, which also are the 3-braids of degree at most 2. Their enumeration in normal form-in an ordering that may seem strange now, but should become familiar soon-is: $1, \mathrm{a}, \mathrm{a} \cdot \mathrm{a}, \mathrm{b}, \mathrm{ba}, \mathrm{ba} \cdot \mathrm{a}, \mathrm{b} \cdot \mathrm{b}, \mathrm{b} \cdot \mathrm{ba}, \mathrm{ab}$,

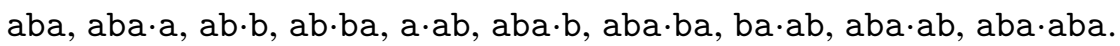

By Lemma 1.5, every divisor of $\Delta_{n}^{d}$ can be expressed as the product of at most $d$ divisors of $\Delta_{n}$, so we certainly have $\# \operatorname{Div}\left(\Delta_{n}^{d}\right) \leqslant(n !)^{d}$ for all $n, d$.

\section{C. The braid ordering.}

Definition 1.7. Let $w$ be a nonempty braid word. We say that $\sigma_{m}$ is the main generator in $w$ if $\sigma_{m}$ or $\sigma_{m}^{-1}$ occurs in $w$, but no $\sigma_{i}^{ \pm 1}$ with $i>m$ does. We say that $w$ is $\sigma$-positive if the main generator occurs only positively in $w$, and similarly it is $\sigma$-negative if that generator occurs negatively. 
A positive nonempty braid word, that is, one that contains no $\sigma_{i}^{-1}$ at all, is $\sigma$ positive, but the inclusion is strict: for instance, $\sigma_{1}^{-1} \sigma_{2}$ is not positive, but it is $\sigma$-positive, as its main generator, namely $\sigma_{2}$, occurs positively (with one $\sigma_{2}$ ) but not negatively (no $\sigma_{2}^{-1}$ ).

The following two properties have received a number of independent proofs [Dehornoy et al. 2002]:

Property A. A $\sigma$-positive braid word does not represent 1 .

Property C. Every braid except 1 can be represented by a $\sigma$-positive word or by a $\sigma$-negative word.

Building on these results, it is straightforward to order the braids:

Definition 1.8. If $x, y$ are braids, we say that $x<y$ holds if the braid $x^{-1} y$ admits at least one $\sigma$-positive representative.

It is clear that the relation $<$ is transitive and compatible with multiplication on the left; Property A implies that $<$ has no cycle and hence is a strict partial order, and Property $\mathrm{C}$ then implies that it is actually a linear order.

As every nonempty positive braid word is $\sigma$-positive, $x \preccurlyeq y$ implies $x \leqslant y$ for all positive braids $x, y$. The converse is not true: $\sigma_{1}$ is not a left divisor of $\sigma_{2}$, but $\sigma_{1}<\sigma_{2}$ holds because $\sigma_{1}^{-1} \sigma_{2}$ is a $\sigma$-positive word.

Example 1.9. The increasing enumeration of the $\operatorname{set} \operatorname{Div}\left(\Delta_{3}\right)$ is

$$
1<\mathrm{a}<\mathrm{b}<\mathrm{ba}<\mathrm{ab}<\mathrm{aba} .
$$

For instance, we have ba $<\mathrm{ab}$, that is, $\sigma_{2} \sigma_{1}<\sigma_{1} \sigma_{2}$ because the quotient, namely $\sigma_{1}^{-1} \sigma_{2}^{-1} \sigma_{1} \sigma_{2}$ (or ABab), also admits the expression $\sigma_{2} \sigma_{1}^{-1}$, a $\sigma$-positive word. Similarly, the reader can check that the increasing enumeration of $\operatorname{Div}\left(\Delta_{3}^{2}\right)$ is the one given in Example 1.6.

Lemma 1.10. The linear ordering < extends the left divisibility ordering $\prec$.

Proof. By definition, $1<\sigma_{i}$ holds for every $i$. As the ordering $<$ is compatible with multiplication on the left, it follows that $x<x \sigma_{i}$ holds for all $i, x$, and, therefore, $x<x y$ holds whenever $y$ is a nontrivial positive braid.

Lemma 1.10 implies that 1 is always the first element of $\left(\operatorname{Div}\left(\Delta_{n}^{d}\right),<\right)$, and $\Delta_{n}^{d}$ is always its last element. A deep result by Laver [1996] shows that, although < is not compatible with right multiplication in general, nevertheless $x<\sigma_{i} x$ always holds, that is, $<$ also extends the right divisibility ordering.

By Property $\mathrm{C}$, every nontrivial braid admits at least one $\sigma$-positive or $\sigma$-negative expression. In general, such a $\sigma$-positive or $\sigma$-negative expression is not unique, but the main generator in such expressions is uniquely defined: 
Lemma 1.11. If a braid $x$ admits a $\sigma$-positive expression, then the main generators in any two $\sigma$-positive expressions of $x$ coincide.

Proof. Assume that $w, w^{\prime}$ are $\sigma$-positive expressions of $x$, and let $\sigma_{m}, \sigma_{m^{\prime}}$ be their main generators. Assume for instance $m<m^{\prime}$. Then $w^{-1} w^{\prime}$ is a $\sigma$-positive word, and it represents the trivial braid 1: this contradicts Property A.

Thus, there will be no ambiguity in referring to the main generator of some nontrivial braid $x$ : this means the main generator in any $\sigma$-positive (or $\sigma$-negative) expression of $x$.

Remark 1.12. Our definition corresponds to the order $<^{\phi}$ of [Dehornoy et al. 2002]. It differs from the one most used in the literature in that the definition of a $\sigma$-position refers to the maximal index rather than the minimal one. Switching from one definition to the other amounts to conjugating by $\Delta_{n}$, that is, to applying the flip automorphism. The results are entirely similar for both versions. However, it is much more convenient to consider the "max" choice here, because it guarantees that $B_{n}^{+}$is an initial segment of $B_{n+1}^{+}$. Using the "min" convention would make the statements in the following sections less natural.

\section{Measuring the ordered $\operatorname{sets}\left(\operatorname{Div}\left(\Delta_{n}^{d}\right),<\right)$}

To investigate the finite ordered sets $\left(\operatorname{Div}\left(\Delta_{n}^{d}\right),<\right)$, and, more generally, the sets $(\operatorname{Div}(z),<$ ) for positive braids $z$, we shall define numerical parameters that reflect their size. The first parameter involves the length of the $\sigma$-positive words that are, in a natural sense defined below, drawn in the Cayley graph of $\Delta_{n}^{d}$. It will be called the complexity of $\Delta_{n}^{d}$, because it is directly connected with the complexity analysis of the handle reduction algorithm of [Dehornoy 1997]. The other parameters involve a filtration of the linear ordering by the $\sigma_{i}$ 's, and they will be called the heights of $\Delta_{n}^{d}$ because they count the jumps of a given height in $\left(\operatorname{Div}\left(\Delta_{n}^{d}\right),<\right)$.

2A. Sigma-positive paths in the Cayley graph. The first parameter we attach to $(\operatorname{Div}(z),<)$ involves the $\sigma$-positive paths in the Cayley graph of $z$.

We recall that the Cayley graph of the group $B_{n}$ with respect to the standard generators $\sigma_{i}$ is a labeled graph: it has the vertex set $B_{n}$ and is such that there exists a $\sigma_{i}$-labeled edge from $x$ to $y$ if and only if $y=x \sigma_{i}$. The Cayley graph of the monoid $B_{n}^{+}$is obtained by restricting the vertices to $B_{n}^{+}$. Note that the Cayley graph of $B_{n}$ (and a fortiori of $B_{n}^{+}$) can be seen as a subgraph of the Cayley graph of $B_{\infty}$.

Definition 2.1. (See Figure 2.) For $z$ a positive braid, we denote by $\Gamma(z)$ the subgraph of the Cayley graph of $B_{\infty}$ obtained by restricting the vertices to $\operatorname{Div}(z)$ and removing the edges do not connect two vertices in $\operatorname{Div}(z)$. 
Because every element of $B_{n}^{+}$is a left divisor of $\Delta_{n}^{d}$ for sufficiently large $d$, the Cayley graph of $B_{n}^{+}$is the union over all $d$ of the graphs $\Gamma\left(\Delta_{n}^{d}\right)$.
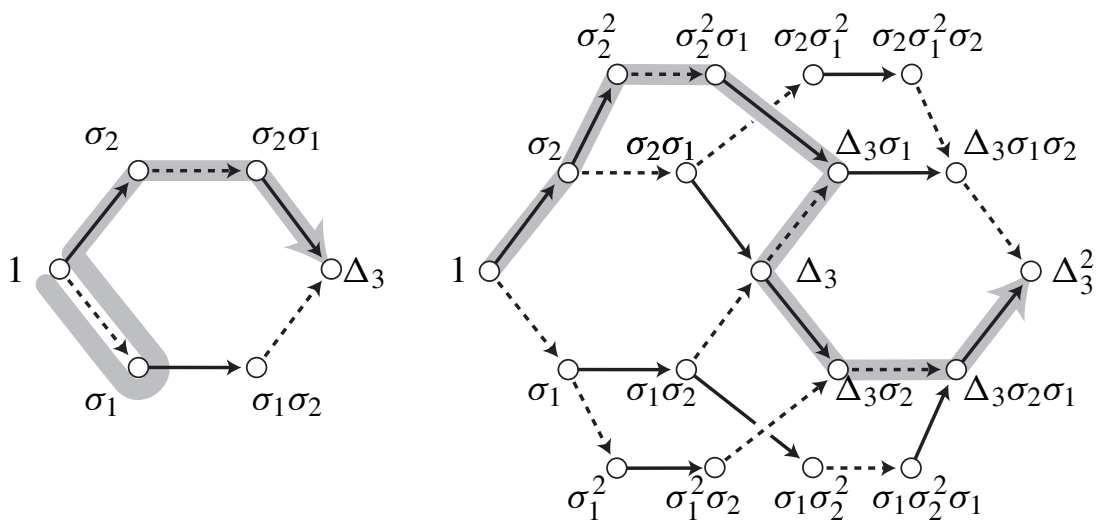

Figure 2. The graphs of $\Gamma\left(\Delta_{3}\right)$ and $\Gamma\left(\Delta_{3}^{2}\right)$; the dotted edges represent $\sigma_{1}$, the plain ones $\sigma_{2}$; observe that the graph of $\Delta_{3}^{2}$ is not planar; in grey: two $\sigma$-positive words traced in the graphs, namely aAbab and bbabAbab (see Lemma 2.3).

A path in the Cayley graph can be specified by its initial vertex and the listed labels of its successive edges, that is, by a braid word. For each $i<n$ and each $x$ in $B_{n}$, there is in $B_{n}$ 's Cayley graph exactly one $\sigma_{i}$-labeled edge leading into $x$ and exactly one other going out of it. Hence, in the complete Cayley graph of $B_{n}$, for each initial vertex $x$ and each $n$-braid word $w$, there is always one path labeled $w$ starting from $x$. When we restrict to some fragment $\Gamma$, this need not be the case, but we do have an unambiguous notion of $w$ being drawn in $\Gamma$ from $x$. Formally:

Definition 2.2. If $\Gamma$ is a subgraph of the Cayley graph of $B_{\infty}$, and $x$ is a vertex in $\Gamma$, we say that a braid word $w$ is drawn from $x$ in $\Gamma$ if, for every prefix $v \sigma_{i}$ (resp. $v \sigma_{i}^{-1}$ ) of $w$, there exists a $\sigma_{i}$-labeled edge starting (resp. finishing) at $x[v]$ in $\Gamma$.

For instance, we can check on Figure 2 that the word $\sigma_{1}^{2}$ is drawn from $\sigma_{2}$ in $\Gamma\left(\Delta_{3}^{2}\right)$, but not in $\Gamma\left(\Delta_{3}\right)$. In algebraic terms,

Lemma 2.3. Assume that $z$ is a positive braid, and $w$ is a braid word. Then $w$ is drawn from $x$ in $\Gamma(z)$ if and only if $x[v] \preccurlyeq z$ holds for each prefix $v$ of $w$.

Proof. The condition is sufficient. Indeed, assume it is satisfied by $w$, and $v \sigma_{i}$ is a prefix of $w$. Then, by hypothesis, $x[v]$ and $x[v] \sigma_{i}$ are left divisors of $z$. Hence are vertices in $\Gamma(z)$, and, therefore, there is a $\sigma_{i}$-labeled edge between $x[v]$ and $x[v] \sigma_{i}$ in $\Gamma(z)$. The argument is similar for a prefix of the form $v \sigma_{i}^{-1}$. Using induction on the length of $w$, we deduce that $w$ is drawn from $x$ in $\Gamma(z)$. 
Conversely, if there is a $w$-labeled path from $x$ in $\Gamma(z)$, then, for each prefix $v$ of $w$, the braid $x[v]$ represents some vertex in $\Gamma(z)$. Hence it's a left divisor of $z$.

For $z$ a positive braid, we shall investigate the $\sigma$-positive words drawn in the graph $\Gamma(z)$. It is clear that, even if $\operatorname{Div}(z)$ is a finite set, arbitrary long words are drawn in $\Gamma(z)$ whenever the latter contains at least 2 vertices, that is, $z$ is not 1 . The example of Figure 2 shows that restricting to $\sigma$-positive words does not change the result: for instance, for each $k$, the word $\left(\sigma_{1} \sigma_{1}^{-1}\right)^{k} \sigma_{2} \sigma_{1} \sigma_{2}$ is a $\sigma$-positive expression of $\Delta_{3}$, and it is drawn in $\Gamma\left(\Delta_{3}\right)$. So we cannot hope for any finite upper bound on the length of the $\sigma$-positive words drawn in $\Gamma(z)$ in general. The situation changes if we concentrate on the main generators, that is, we forget about the generators with nonmaximal index.

Lemma 2.4. Assume that $\Gamma$ is subgraph of the Cayley graph of $B_{\infty}$, and $w$ is a $\sigma$-positive word drawn in $\Gamma(z)$. Then the number of occurrences of the main generator in $w$ is at most the number of nonterminal vertices in $\Gamma$.

Proof. Assume that $w$ is drawn from $x$ in $\Gamma$. Let $\sigma_{m}$ be the main generator in $w$. As there is at most one $\sigma_{m}$-labeled edge starting from each vertex of $\Gamma$, it suffices to show that the number of $\sigma_{m}$ 's in $w$ is bounded above by the number of $\sigma_{m}$-edges in $\Gamma$. Hence, it suffices to show that the path $\gamma$ associated with $w$ cannot cross the same $\sigma_{m}$-edge twice. Now assume that some $\sigma_{m}$-edge starts from the vertex $y$, and that $\gamma$ crosses this edge twice. This means that $\gamma$ contains a loop from $y$ to $y$. Let $v$ be the subword of $w$ labeling that loop. By construction, $v$ begins with $\sigma_{m}$, it contains no $\sigma_{m}^{-1}$ and no $\sigma_{i}^{ \pm 1}$ with $i>m$ as it is a subword of $w$, and it represents the braid 1 as it labels a loop in the Cayley graph of $B_{\infty}$ : this means that $v$ is a $\sigma$-positive word representing 1, which contradicts Property A.

Lemma 2.4 applies in particular to every graph $\Gamma(z)$ in which $z$ is a positive braid. We can introduce our first parameter measuring the size of the ordered set $(\operatorname{Div}(z),<)$ :

Definition 2.5. (See Figure 2.) When $z$ is a positive braid with main generator $\sigma_{m}$, the complexity $c(z)$ of $z$ is defined to be the maximal number of $\sigma_{m}$ 's in a $\sigma$-positive word drawn in $\Gamma(z)$.

Example 2.6. The word $\sigma_{2} \sigma_{1} \sigma_{2}$ is a $\sigma$-positive word drawn from 1 in $\Gamma\left(\Delta_{3}\right)$, and it contains two $\sigma_{2}$ 's. Hence we have $c\left(\Delta_{3}\right) \geqslant 2$. Actually, it is not hard to obtain the exact value $c\left(\Delta_{3}\right)=2$. Indeed, if a $\sigma$-positive path $\gamma$ contains the two $\sigma_{2}$-edges starting from 1 and $\sigma_{1} \sigma_{2}$, it cannot come back to $\sigma_{2}$ without crossing the third $\sigma_{2}$-edge; and if $\gamma$ contains the $\sigma_{2}$-edge that starts from $\sigma_{1}$, it can never come back to 1 or to $\sigma_{2} \sigma_{1}$ and therefore contains at most one $\sigma_{2}$-edge. As we have $\Delta_{3}^{d}=\left(\sigma_{2} \sigma_{1} \sigma_{2}\right)^{d}$, we deduce $c\left(\Delta_{3}^{d}\right) \geqslant 2 d$ for every $d$. This value is certainly not 
optimal: Figure 2 contains five $\sigma_{2}$ 's, proving $c\left(\Delta_{3}^{2}\right) \geqslant 5$. The exact value here is 6 , and, more generally, we have $c\left(\Delta_{n}^{d}\right)=2^{d+1}-2$, as will be seen in Section 3 .

Remark 2.7. Restricting to $\sigma$-positive words drawn in $\Gamma(z)$ is essential: for instance, for each $k$, we have

$$
\Delta_{3}=\sigma_{2}^{k+1} \sigma_{1} \sigma_{2} \sigma_{1}^{-k},
$$

a $\sigma$-positive word containing $k+2$ letters $\sigma_{2}$. Now, for $k \geqslant 1$, the word involved in (2-1) is not drawn in $\Gamma\left(\Delta_{3}^{1}\right)$, because its prefix $\sigma_{2}^{2}$ is not. Thus the parameter $c(z)$ does involve the left divisors of $z$.

Directly applying Lemma 2.4 gives:

Proposition 2.8. Every positive braid has a finite complexity; more precisely, for $z$ of length $\ell$ in $B_{n}^{+}$with $n \geqslant 3$, we have $c(z) \leqslant(n-1)^{\ell}$.

Proof. The number of nonterminal vertices in $\Gamma(z)$, that is, the number of proper left divisors of $z$, is at most $1+(n-1)+(n-1)^{2}+\cdots+(n-1)^{\ell-1}$.

As the length of any positive expression of $\Delta_{n}$ is $n(n-1) / 2$, we obtain in particular for all $n, d$

$$
c\left(\Delta_{n}^{d}\right) \leqslant(n-1)^{d n(n-1) / 2} .
$$

Before going further, we observe that, in defining the complexity of $z$, we can restrict to decompositions of $z$, that is, instead of considering paths starting and finishing at arbitrary vertices, we can restrict to paths going from 1 to $z$ :

Lemma 2.9. Assume that $z$ is a positive braid with main generator $\sigma_{m}$. Then $c(z)$ is the maximal number of $\sigma_{m}$ 's in any $\sigma$-positive decomposition of $z$ drawn in $\Gamma(z)$.

Proof. Let $c^{\prime}(z)$ be the number involved in the above statement. Clearly we have $c^{\prime}(z) \leqslant c(z)$. Conversely, assume that $w$ is drawn in $\Gamma(z)$ from $x$, and that the $w$-labeled path starting at $x$ finishes at $y$. Let $u$ be a positive expression of $x$, and $v$ be a positive expression of $y^{-1} z$. The latter exists as, by hypothesis, $y$ is a left divisor of $z$. Then $u w v$ is a $\sigma$-positive decomposition of $z$ drawn in $\Gamma(z)$. Hence we have $c^{\prime}(z) \geqslant c(z)$.

Remark 2.10. We call Property A* the statement that all numbers $c\left(\Delta_{n}^{d}\right)$ are finite. Above, we derived Property $A^{*}$ from Property A. The two properties are actually equivalent, that is, we can also deduce Property A from Property $\mathrm{A}^{*}$. For that, assume that some $\sigma$-positive braid word $w$ represents 1 . The word $w$ may involve negative letters. We must find a vertex $x$ that begins a path labeled $w$ in some $\Gamma\left(\Delta_{n}^{d}\right)$. Let $\sigma_{m}$ be the main generator in $w$. The word $w$ has finitely many prefixes, say $w_{0}, \ldots, w_{\ell}$. By Garside theory, each word $w_{i}$ is equivalent to one the form 
$u_{i}^{-1} v_{i}$, with $u_{i}, v_{i}$ positive. Let $x$ be the least common left multiple of the positive braids

$$
\left[u_{0}\right], \ldots,\left[u_{\ell}\right] .
$$

For each $i$, the braid $x\left[w_{i}\right]$ is positive. Moreover, there exist $n$ and $d$ such that

$$
x\left[w_{0}\right], \ldots, x\left[w_{\ell}\right]
$$

are all divisors of $\Delta_{n}^{d}$. Thus the word $w$ is drawn from $x$ in $\Gamma\left(\Delta_{n}^{d}\right)$, and the associated path is a loop around $x$. It follows that $w^{k}$ is drawn in $\Gamma\left(\Delta_{n}^{d}\right)$ from $x$ for each $k$. By construction, $w^{k}$ contains at least $k$ generators $\sigma_{m}$. Hence $c\left(\Delta_{n}^{d}\right)$ cannot be finite.

2B. Connection with handle reduction. Handle reduction [Dehornoy 1997] is an algorithmic solution to the word problem of braids that relies on the braid ordering. It is the most efficient method today. The method converges, and the argument in [Dehornoy 1997] shows the complexity upper bound to be exponential in the input word length, an estimate seemingly very far from sharp.

Each step of handle reduction involves a specific generator $\sigma_{i}$, and, for an induction, the point is to obtain an upper bound on the reduction steps involving the main generator. The latter will naturally be called the main reduction steps. The connection between handle reduction and the complexity as defined above relies on the following technical result:

Lemma 2.11 [Dehornoy 1997]. Assume that $z$ is a positive braid with main generator $\sigma_{m}$ and that $w$ is drawn in $\Gamma(z)$. Then, for each sequence of handle reductions from $w$-that is, each sequence $\vec{w}$ with $w_{0}=w$ such that $w_{k}$ is obtained by reducing one handle from $w_{k-1}$ for each $k$-there exists a witness-word $u$ that is $\sigma$-positive, drawn in $\operatorname{Div}(z)$, and such that the number of $\sigma_{m}$ 's in $u$ is the number of main reductions in $\vec{w}$.

It follows that the number of main reduction steps in any sequence of handle reductions starting with a word drawn in $\Gamma(z)$ is bounded above by $c(z)$. In particular, if we start with an $n$ strand braid word $w$ of length $\ell$, then it is easy to show that $w$ is drawn in $\Gamma\left(\Delta_{n}^{\ell}\right)$, and, applying the upper bound of Equation (2-2), we deduce the upper bound on the number of possible main reductions from $w$, and it is exponential in $\ell$.

A natural way to improve this coarse upper bound would be to determine $c\left(\Delta_{n}^{d}\right)$ more precisely. This will be done in Section 3 below. However, the explicit formulas show that, for $n \geqslant 3$, the growth in $d$ really is exponential, thus dashing any hopes of proving a polynomial upper bound for the number of reduction steps by this approach. 
2C. A filtration of the braid ordering. We now introduce new numerical parameters for the ordered sets $(\operatorname{Div}(z),<)$. These numbers connect with a natural filtration of the ordering $<$, using an increasing sequence of partial orderings.

By Lemma 1.11, the index of the main generator of a nontrivial braid is well defined. We can use this index to measure the height of the jump between two braids $x, y$ satisfying $x<y$ :

Definition 2.12. For $x, y$ in $B_{\infty}$ and $r \geqslant 1$, we say that $x<_{r} y$ holds or, equivalently, that $(x, y)$ is an $r$-jump, if $x^{-1} y$ admits a $\sigma$-positive expression in which the main generator is $\sigma_{m}$ with $m \geqslant r$.

Lemma 2.13. For each $r \geqslant 1$, the relation $<_{r}$ is a strict partial order that refines $<$; the relation $<_{1}$ coincides with $<$, and $r \leqslant q$ implies that $<_{q}$ refines $<_{r}$.

Proof. That $<_{r}$ is transitive follows because the concatenation of a $\sigma$-positive word with main generator $\sigma_{m}$ and a $\sigma$-positive word with main generator $\sigma_{m^{\prime}}$ is a $\sigma$-positive word with main generator $\sigma_{\max \left(m, m^{\prime}\right)}$.

In the sequel, we consider the $<_{r}$-chains included in $\operatorname{Div}(z)$, and their length:

Definition 2.14. For $z$ a positive braid and $r \geqslant 1$, we define the $r$-height $h_{r}(z)$ of $z$ to be the maximal length of $\mathrm{a}<_{r}$-chain included in $\operatorname{Div}(z)$.

Before giving examples, we observe the connection between $h_{r}(z)$ and the increasing enumeration of the $\operatorname{set} \operatorname{Div}(z)$ :

Lemma 2.15. Let $z$ be a positive braid and $r \geqslant 1$. Then $h_{r}(z)-1$ is the number of $r$-jumps in the increasing enumeration of $(\operatorname{Div}(z),<)$.

Proof. If the number of $r$-jumps in the increasing enumeration of $\operatorname{Div}(z)$ is $N_{r}-1$, we can extract from $\operatorname{Div}(z)$ a $<_{r}$-chain of length $N_{r}$. Conversely, assume that $\left(y_{0}, \ldots y_{N_{r}}\right)$ is a $<_{r}$-chain in $\operatorname{Div}(z)$. Let $z_{0}<\ldots<z_{N}$ be the increasing enumeration of $\operatorname{Div}(z)$. As $<_{r}$ refines $<$, there exists an increasing function $f$ of $\left\{0, \ldots, N_{r}\right\}$ into $\{0, \ldots, N\}$ such that $y_{i}=z_{f(i)}$ holds for every $i$. Now the hypothesis $z_{f(i)}<_{r} z_{f(i+1)}$ implies that there exists at least one $r$-jump between $z_{f(i)}$ and $z_{f(i+1)}$. Indeed, by Lemma 1.11, it is impossible that a concatenation of $m$ jumps with $m<r$ results in a $r$-jump. So the number of $r$-jumps in $\left(z_{0}, \ldots, z_{N}\right)$ is at least $N_{r}$.

In other words, to determine $h_{r}(z)$, there is no need to consider arbitrary chains: it is enough to consider the maximal chain obtained by enumerating $\operatorname{Div}(z)$ exhaustively.

Example 2.16. Refining the increasing enumeration of $\operatorname{Div}\left(\Delta_{3}\right)$ of Example 1.9 by indicating for each step the height of the corresponding jump, we obtain:

$$
1<_{1} \mathrm{a}<_{2} \mathrm{~b}<_{1} \text { ba }<_{2} \mathrm{ab}<_{1} \Delta_{3},
$$


where we recall a, b, ... stand for $\sigma_{1}, \sigma_{2}, \ldots$ For instance, (ba, ab) is a 2-jump, because we have in $(\mathrm{ba})^{-1}(\mathrm{ab})=\mathrm{ABab}=\mathrm{AabA}=\mathrm{bA}$ a $\sigma$-positive decomposition with main generator $\sigma_{2}$. The number of 1-jumps in (2-3), that is, the number of symbols $<_{r}$ with $r \geqslant 1$, is 5 , while the number of 2-jumps is 2, so, by Lemma 2.15, we deduce $h_{1}\left(\Delta_{3}\right)=6$ and $h_{2}\left(\Delta_{3}\right)=3$. Similarly, we obtain for $\Delta_{3}^{2}$

$1<_{1} \mathrm{a}<_{1}$ aa $<_{2} \mathrm{~b}<_{1}$ ba $<_{1}$ baa $<_{2} \mathrm{bb}<_{1}$ bba $<_{2}$ ab $<_{1}$ aba $<_{1}$ abaa $<_{2}$ abb $<_{1}$ abba $<_{2}$ aab $<_{1}$ aaba $<_{1}$ aabaa $<_{2}$ baab $<_{1}$ baaba $<_{1}$ baabaa, leading to $h_{1}\left(\Delta_{3}^{2}\right)=19$ and $h_{2}\left(\Delta_{3}^{2}\right)=7$.

Proposition 2.17. (i) For every braid $z$ in $B_{n}^{+}$, we have

$$
h_{1}(z)=\# \operatorname{Div}(z) \geqslant h_{2}(z) \geqslant \cdots \geqslant h_{n}(z)=1 .
$$

(ii) For all positive braids $z, z^{\prime}$ and $r \geqslant 1$, we have

$$
h_{r}\left(z z^{\prime}\right) \geqslant h_{r}(z)+h_{r}\left(z^{\prime}\right) .
$$

Proof. (i) A $<{ }_{1}$-chain is simply a $<$-chain. Hence every subset of $\operatorname{Div}(z)$ gives such a chain. So the maximal $<_{1}$-chain in $\operatorname{Div}(z)$ is $\operatorname{Div}(z)$ itself, and $h_{1}(z)$ is the cardinality of $\operatorname{Div}(z)$.

On the other hand, no $<_{n}$-chain in $B_{n}^{+}$has length more than 1 , as the main generator of a $\sigma$-positive $n$-strand braid word cannot be $\sigma_{n}$ or any generator above it. Thus $h_{n}(z)$ is 1 .

Then, for $q \leqslant r$, every $<_{r}$-chain is a $<_{q}$-chain, which implies $h_{r}(z) \geqslant h_{q}(z)$.

Point (ii) is obvious, as the concatenation of two $<_{r}$-chains is a $<_{r}$-chain.

From (2-4) we deduce $h_{r}\left(z^{d}\right) \geqslant d \cdot h_{r}(z)$ for all $r, z$. By Lemma 1.5, every divisor of $\Delta_{n}^{d}$ can be decomposed as the product of at most $d$ divisors of $\Delta_{n}$. There are $n !$ such divisors, so we obtain the (coarse) bounds

$$
d \cdot h_{r}\left(\Delta_{n}\right) \leqslant h_{r}\left(\Delta_{n}^{d}\right) \leqslant(n !)^{d},
$$

for all $r, n, d$. Better estimates will be given below.

Remark 2.18. Instead of restricting to subsets of $B_{\infty}$ of the form $\operatorname{Div}(z)$, we can define the complexity and the $r$-height for every (finite) set of braids $X$. Most of the general results extend, but, when $X$ is not closed under left division, nothing can be said about the number of $\sigma_{r}$ 's involved in an $r$-jump. Considering such an extension is not useful here.

2D. Connection with the complexity. We shall now connect the complexity $c(z)$ with the numbers $h_{r}(z)$ just defined. The result is simple: 
Proposition 2.19. For $z$ a positive braid with main generator $\sigma_{m}$, we have

$$
c(z)=h_{m}(z)-1 .
$$

In particular, for $n \geqslant 2$ and $d \geqslant 0$, we have

$$
c\left(\Delta_{n}^{d}\right)=h_{n-1}\left(\Delta_{n}^{d}\right)-1 .
$$

One inequality is easy:

Lemma 2.20. For $z$ a positive braid with main generator $\sigma_{m}$, we have $c(z) \leqslant$ $h_{m}(z)-1$.

Proof. The argument is reminiscent of the one used for Lemma 2.15 but requires a little more care. Assume that $w$ is a $\sigma$-positive word drawn in $\Gamma(z)$ from $x$ containing $N_{m}$ occurrences of $\sigma_{m}$. By Lemma 2.9, we can assume $x=1$ without loss of generality. Let $z_{0}<z_{1}<\ldots<z_{N}$ be the increasing enumeration of $\operatorname{Div}(z)$. By definition, all prefixes of $w$ represent divisors of $z$, so, letting $\ell$ be the length of $w$, there exists a mapping $f:\{0, \ldots, \ell\} \rightarrow\{0, \ldots, N\}$ such that, for each $k$, the length $k$ prefix of $w$ represents $z_{f(k)}$. By construction, we have $f(0)=0$ and $f(\ell)=N$.

The difference from Lemma 2.15 is that $f$ need not be increasing. Now, let $p_{1}, \ldots, p_{N_{m}}$ be the $N_{m}$ positions in $w$ where the generator $\sigma_{m}$ occurs, completed with $p_{0}=0$. Then, in the prefix of $w$ of length $p_{1}$, that is, in the subword of $w$ corresponding to positions from $p_{0}+1$ to $p_{1}$, there is one $\sigma_{m}$, plus letters $\sigma_{i}^{ \pm 1}$ with $i<m$ (Figure 3). This subword is therefore $\sigma$-positive. Hence we must have $z_{f\left(p_{0}\right)}<z_{f\left(p_{1}\right)}$, which requires $f\left(p_{0}\right)<f\left(p_{1}\right)$. Moreover, the quotient $z_{f\left(p_{0}\right)}^{-1} z_{f\left(p_{1}\right)}$ is a braid that admits at least one $\sigma$-positive expression containing $\sigma_{m}$, and hence $z_{f\left(p_{0}\right)}<_{m} z_{f\left(p_{1}\right)}$. Now the same is true between $f\left(p_{1}\right)$ and $f\left(p_{2}\right)$, etc. Hence the number of $m$-jumps in the increasing enumeration of $\operatorname{Div}(z)$ is at least $N_{m}$, that is, we have $h_{m}(z) \geqslant N_{m}+1$.

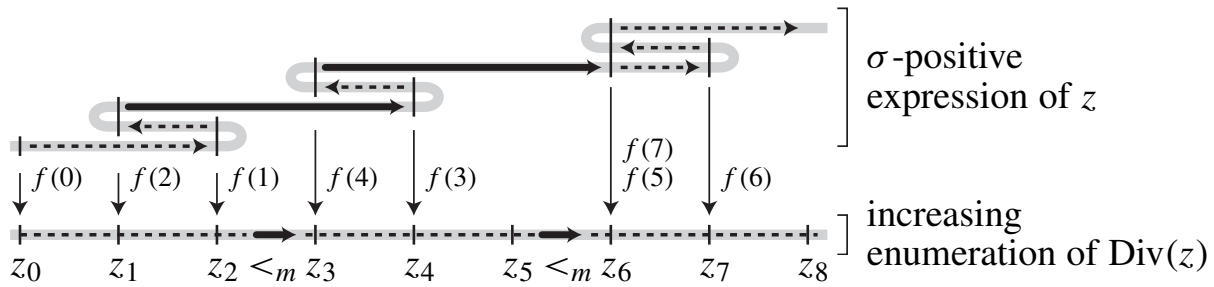

Figure 3. Proof of Lemma 2.20. The main generator $\sigma_{m}$ corresponds to the bold arrow: the function $f$ need not be increasing, but the projection of a bold arrow upstairs must include at least one bold arrow downstairs, that is, at least one $m$-jump. 
It remains to prove the second inequality in Proposition 2.19, that is, to prove that, if $z$ is a positive $n$-braid satisfying $h_{m}(z)=N+1$, then $z$ admits a $\sigma$-positive expression containing $N$ generators $\sigma_{m}$. The problem is as follows: if $z$ is a positive braid and $x, y$ are left divisors of $z$ satisfying $x<y$, then, by definition, the quotient $x^{-1} y$ admits some $\sigma$-positive expression $w$, but nothing a priori guarantees that $w$ be drawn in $\Gamma(z)$. In other words, we might have $x<y$ but no $\sigma$-positive witness for this inequality inside $\operatorname{Div}(z)$. It turns out this cannot happen, but the proof requires a rather delicate argument.

Proposition 2.21. Let $z$ be a positive braid. Then, for all $x, y$ in $\operatorname{Div}(z)$, the following are equivalent:

(i) The relation $x<y$ holds, that is, there exists a $\sigma$-positive path from $x$ to $y$ in the Cayley graph of $B_{\infty}$;

(ii) There exists a $\sigma$-positive path from $x$ to $y$ in the Cayley graph of $B_{n}$;

(iii) There exists a $\sigma$-positive path from $x$ to $y$ in $\Gamma(z)$.

Proof. Clearly (iii) implies (ii), which in turn implies (i). We shall prove that (i) implies (iii) — and thus reprove that (i) implies (ii), which was first proved in [Larue 1994] — by using the handle reduction method of [Dehornoy 1997; Dehornoy et al. 2002]. The problem is to prove that, among all $\sigma$-positive paths connecting $x$ to $y$ in the Cayley graph of $B_{\infty}$, at least one is drawn in $\Gamma(z)$.

Now, let $u, v$ be positive words representing $x$ and $y$. Then the word $u^{-1} v$ represents $x^{-1} y$, and, by hypothesis, it is drawn in $\Gamma(z)$ from $x$. Handle reduction transforms a braid word into equivalent words and eventually produces a $\sigma$-positive word if it exists. It is proved in [Dehornoy 1997] that, for every $n$ strand braid word $w$, there exists a finite fragment $\Gamma_{w}$ of the Cayley graph of $B_{n}^{+}$and a vertex $x_{w}$ of $\Gamma_{w}$ such that $w$ and all words obtained from $w$ by handle reduction are drawn from $x_{w}$ in $\Gamma_{w}$. Moreover, when $w$ has the form $u^{-1} v$ with $u, v$ positive, then all vertices in $\Gamma_{w}$ are the left divisors of the least common right multiple of the braids represented by $u$ and $v$, here $x$ and $y$, while $x_{w}$ is the braid represented by $u$, that is, $x$. As $x$ and $y$ are divisors of $z$, so is their least common right multiple, and the graph $\Gamma_{w}$ is included in $\Gamma(z)$. It follows that every word obtained from $u^{-1} v$ using handle reduction is drawn from $x$ in $\Gamma(z)$. The termination of handle reduction guarantees that, among these words, at least one is $\sigma$-positive, so (iii) follows.

A direct application of Proposition 2.21 is the existence of $\sigma$-positive quotient sequences drawn in the Cayley graph. The definition is as follows:

Definition 2.22. Assume that $z$ is a positive braid and $X$ is a subset of $\operatorname{Div}(z)$. Let $x_{0}<\ldots<x_{N}$ be the increasing enumeration of $X$. We say that a sequence of words $\vec{w}=\left(w_{1}, \ldots, w_{N}\right)$ is a quotient sequence for $X$ if, for each $k$, the word $w_{k}$ is an expression of $x_{k-1}^{-1} x_{k}$ for each $k$. We say that $\vec{w}$ is $\sigma$-positive if every entry in 
$\vec{w}$ is $\sigma$-positive, and that $\vec{w}$ is drawn in $\Gamma(z)$ (from $x_{0}$ ) if $w_{k}$ is drawn from $x_{k-1}$ in $\Gamma(z)$ for each $k$.

Corollary 2.23. Assume that $z$ is a positive braid. Then every subset of $\operatorname{Div}(z)$ admits a $\sigma$-positive quotient sequence drawn in $\Gamma(z)$.

Example 2.24. (Figure 4) By computing the successive quotients in the increasing enumeration of $\operatorname{Div}\left(\Delta_{3}^{2}\right)$ given in Example 1.9, we easily find that

$$
\text { (a, a, AAb, a, a, AAb, a, AAb, a, a, bAA, a, bAA, a, a, bAA, a, a) }
$$

is a $\sigma$-positive quotient sequence for $\operatorname{Div}\left(\Delta_{3}^{2}\right)$ drawn in $\Gamma\left(\Delta_{3}^{2}\right)$. This sequence turns out to be the unique sequence with the above properties, but this uniqueness is specific to the case of 3-braids (see Figure 8 below).

We can now easily complete the proof of Proposition 2.19:

Proof of Proposition 2.19. Let $\left(z_{0}, \ldots, z_{N}\right)$ be the $<$-increasing enumeration of $\operatorname{Div}(z)$. By Corollary 2.23, there exists a $\sigma$-positive quotient sequence $\vec{w}$ for $\operatorname{Div}(z)$ that is drawn in $\Gamma(z)$. Let $w=w_{1} \ldots w_{N}$. By construction, $w$ is a $\sigma$-positive word drawn in $\Gamma(z)$, and the number of occurrences of the main generator $\sigma_{m}$ in $w$ is (at least) the number of $m$-jumps in $\left(z_{0}, \ldots, z_{N}\right)$. So we have $c(z) \geqslant h_{m}(z)-1$. Invoking Lemma 2.20 completes the proof.

Remark 2.25. Assume that $\vec{w}$ is a $\sigma$-positive quotient sequence for $\operatorname{Div}(z)$, and $\sigma_{m}$ is the main generator occurring in $\vec{w}$. Then each word $w_{i}$ contains zero or one letter $\sigma_{m}$. Indeed, if $w_{i}$ contained two $\sigma_{m}$ 's or more, then the vertex reached after the first $\sigma_{m}$ ought to lie in the open <-interval determined by two successive entries of $\vec{z}$, and the latter is empty by construction since all elements of $\operatorname{Div}(z)$ occur in $\vec{z}$.

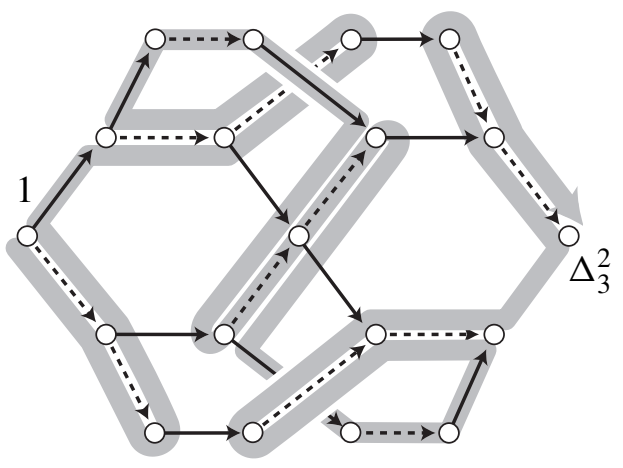

Figure 4. The increasing enumeration of the divisors of $\Delta_{3}^{2}$, and a $\sigma$-positive quotient sequence drawn in $\Gamma\left(\Delta_{3}^{2}\right)$ : the associated path visits every vertex, and is labeled aaAAbaaAAbabAAa aAAbabAAaabAAaa; it crosses $6 \sigma_{2}$-edges (and no $\sigma_{2}^{-1}$ ). 


\section{A decomposition result for $(\operatorname{Div}(z),<)$}

In this section, we establish a structural result describing $\left(\operatorname{Div}\left(\Delta_{n}^{d}\right),<\right)$ as the concatenation of $c\left(\Delta_{n}^{d}\right)+1$ intervals isomorphic to subsets of $\left(\operatorname{Div}\left(\Delta_{n-1}^{d}\right),<\right)$. We deduce an explicit formula connecting $h_{r}\left(\Delta_{n}^{d}\right)$ with the number of braids in $\operatorname{Div}\left(\Delta_{n}^{d}\right)$ whose $d$-th factor is right divisible by $\Delta_{r}$, which in turn enables us to finish computing $c\left(\Delta_{n}^{d}\right)$ and $h_{r}\left(\Delta_{n}^{d}\right)$ for small values of $r, n$ and $d$.

3A. B $\boldsymbol{B}_{\boldsymbol{r}}$-classes. To analyze the linearly ordered sets $\left(\operatorname{Div}\left(\Delta_{n}^{d}\right),<\right)$, and, more generally, $(\operatorname{Div}(z),<)$ for $z$ a positive braid, we introduce convenient partitions. As $B_{r}$ is a group for each $r$, it is clear that the relation $x^{-1} y \in B_{r}$ defines an equivalence relation on (positive) braids, so we may put:

Definition 3.1. For $r \geqslant 1$ and $x, y$ in $B_{\infty}^{+}$, we say that $x$ and $y$ are $B_{r}$-equivalent if $x^{-1} y$ belongs to $B_{r}$.

By construction, $B_{r}$-equivalence is compatible with multiplication on the left. In the sequel, we consider the restriction of $B_{r}$-equivalence to finite subsets of $B_{\infty}^{+}$ of the form $\operatorname{Div}(z)$, that is, we use $B_{r}$-equivalence to partition $\operatorname{Div}(z)$ into subsets, naturally called $B_{r}$-classes.

Example 3.2. As $B_{1}$ is trivial, $B_{1}$-equivalence is equality, and so, therefore, the $B_{1}$-classes are singletons. On the other hand, any two elements of $B_{n}$ are $B_{r^{-}}$ equivalent for each $r \geqslant n$, so, for $z$ in $B_{n}^{+}$, there is only one $B_{r}$-class for $r \geqslant n$, and the only interesting relations arise for $1<r<n$. For instance, $\operatorname{Div}\left(\Delta_{3}\right)$ contains three $B_{2}$-classes, while $\operatorname{Div}\left(\Delta_{3}^{2}\right)$ contains seven of them (Figure 5).

Saying that there is an $r$-jump between two braids $x$ and $y$ means that $x^{-1} y$ is $\sigma$-positive and does not belong to $B_{r}$, so, for $x<y$, we have the equivalence

$$
\left(x, y \text { are not } B_{r} \text {-equivalent }\right) \Longleftrightarrow\left(\begin{array}{c}
\text { there is a } r \text {-jump between } \\
\text { between } x \text { and } y
\end{array}\right) \text {. }
$$
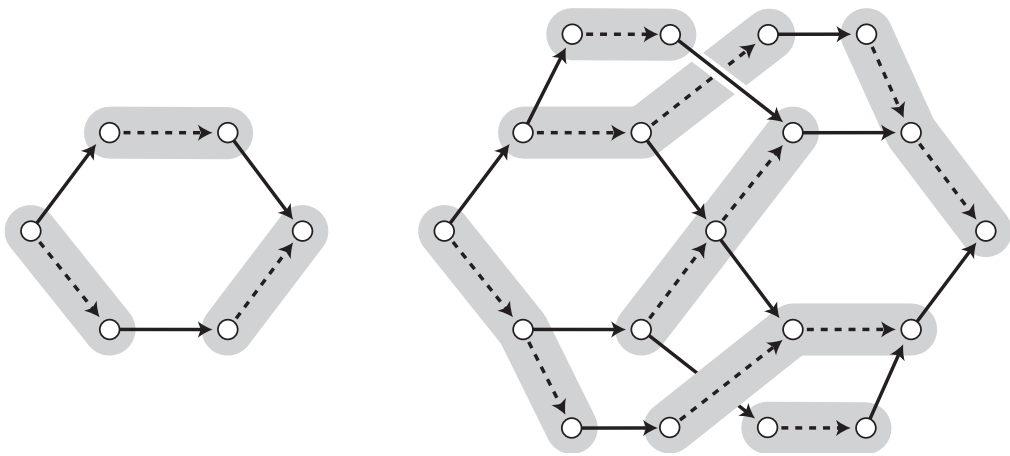

Figure 5. The $B_{2}$-classes in $\operatorname{Div}\left(\Delta_{3}\right)$ and $\operatorname{Div}\left(\Delta_{3}^{2}\right)$. 
Lemma 3.3. Assume that $z$ is a positive braid. Then, each $B_{r}$-class in $\operatorname{Div}(z)$ is an interval for $<$ and there is an $r$-jump between each $B_{r}$-class and the next one.

Proof. Assume $x<y \in \operatorname{Div}(z)$. By (3-1), if $x$ and $y$ are not $B_{r}$-equivalent, there is an $r$-jump between $x$ and $y$ and hence also between $x$ and any element of $\operatorname{Div}(z)$ above $y$. Thus no such element may be $B_{r}$-equivalent to $x$. This implies that each $B_{r}$-class is an $<$-interval.

Corollary 3.4. For each $r \geqslant 1$, the number of $B_{r}$-classes in $\operatorname{Div}(z)$ is $h_{r}(z)$.

Proof. By (3-1), there is no $r$-jump between two elements of the same $B_{r}$-class, and there is one between two elements not in the same $B_{r}$-class. Thus the number of $B_{r}$-classes is the number of $r$-jumps in the $<$-increasing enumeration of $\operatorname{Div}(z)$ augmented by 1 . Hence, by Lemma 2.15 , it is $h_{r}(z)$.

With $B_{r}$-equivalence, we can partition $(\operatorname{Div}(z),<)$ into finitely many subintervals. The interest of this partition is that we can describe $B_{r}$-classes rather precisely and, typically, connect them with subsets of $B_{r}$. In particular, this will allow for connecting the ordered sets $\left(\operatorname{Div}\left(\Delta_{n}^{d}\right),<\right)$ with the sets $\left(\operatorname{Div}\left(\Delta_{n-1}^{d}\right),<\right)$.

Proposition 3.5 (Figure 6). Assume $z \in B_{\infty}^{+}$and $r \geqslant 1$. Let $C$ be a $B_{r}$-class in $\operatorname{Div}(z)$, and let $a, b$ be its <-extremal elements. Then a divides every element of $C$ on the left, and the left translation by a establishes an isomorphism between $\left(\operatorname{Div}\left(a^{-1} b\right), \preccurlyeq,<\right)$ and $(C, \preccurlyeq,<)$. In particular, $(C, \preccurlyeq)$ is a lattice.

Proof. By Lemma 3.3, $C$ is the $<$-interval determined by $a$ and $b$, that is, we have

$$
C=\{x \in \operatorname{Div}(z) ; a<x<b\} .
$$

We know that $\operatorname{Div}(z)$ is a lattice with respect to left divisibility: any two elements $x, y$ of $\operatorname{Div}(z)$ admit a greatest common left divisor, here denoted $\operatorname{gcd}(x, y)$, and a least common right multiple, denoted $\operatorname{lcm}(x, y)$. Firstly, we claim that $C$ is a lattice with respect to left divisibility, that is, the left gcd and the right lcm of two elements of $C$ lie in $C$. So assume $x, y \in C$. Let $x_{0}, y_{0}$ be defined by $x=\operatorname{gcd}(x, y) x_{0}$ and $y=\operatorname{gcd}(x, y) y_{0}$. The hypothesis that $x^{-1} y$ belongs to $B_{r}$ implies that there exist $x_{1}, y_{1}$ in $B_{r}^{+}$satisfying $x^{-1} y=x_{1}^{-1} y_{1}$. By definition of the gcd, there must exist a positive braid $z_{1}$ satisfying $x_{1}=z_{1} x_{0}$ and $y_{1}=z_{1} y_{0}$. Because $z_{1}$ is positive, $x_{1} \in B_{r}^{+}$implies $x_{0} \in B_{r}^{+}$, and hence $\operatorname{gcd}(x, y) \in C$. As for the $\mathrm{lcm}$, the conjunction of $x=\operatorname{gcd}(x, y) x_{0}$ and $y=\operatorname{gcd}(x, y) y_{0}$ implies

$$
\operatorname{lcm}(x, y)=\operatorname{gcd}(x, y) \operatorname{lcm}\left(x_{0}, y_{0}\right) .
$$

As $x_{0}, y_{0} \in B_{r}^{+}$implies $\operatorname{lcm}\left(x_{0}, y_{0}\right) \in B_{r}^{+}$, we deduce $\operatorname{lcm}(x, y) \in C$.

As $C$ is finite, it follows that $C$ admits a global gcd. Because the linear ordering $\leqslant$ extends the partial divisibility ordering $\preccurlyeq$, this global gcd must be the $<-$ minimum $a$ of $C$. Symmetrically, $C$ admits a global $\mathrm{lcm}$, which must be the 


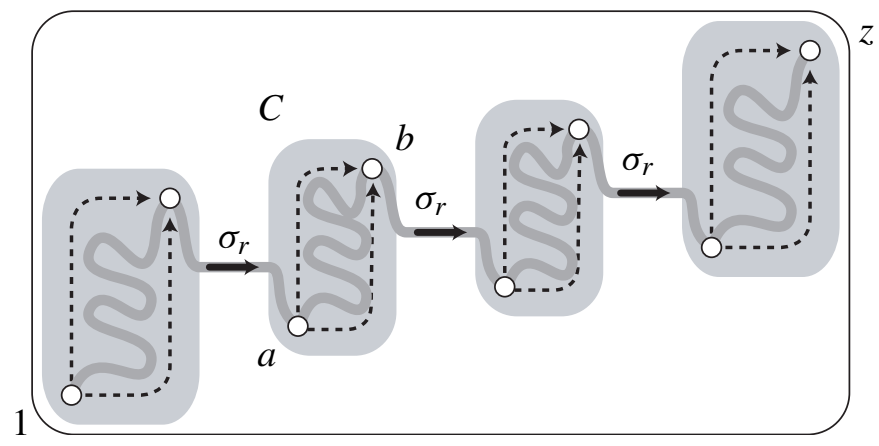

Figure 6. Decomposition of $(\operatorname{Div}(z),<)$ into $B_{r}$-classes: each class $C$ is a lattice with respect to divisibility; the increasing enumeration of $\operatorname{Div}(z)$ exhausts the first class, then jumps to the next one by an $r$-jump, etc. The number of classes is $h_{r}(z)$.

$<$-maximum $b$. So, at this point, we know that $a$ is a left divisor of every element in $C$, and $b$ is a right multiple of each such element, that is, we have

$$
C \subseteq\left\{x \in B_{\infty}^{+} ; a \preccurlyeq x \preccurlyeq b\right\} .
$$

Moreover, $a \preccurlyeq x \preccurlyeq b$ implies $a \leqslant x \leqslant b$. Hence $x \in C$, and so the inclusion in (3-2) is an equality.

Now, put $F(x)=a x$ for $x$ in $\operatorname{Div}\left(a^{-1} b\right)$. As $B_{\infty}^{+}$is left cancellative, $F$ is injective. Moreover, for $x$ a positive braid, $x \preccurlyeq a^{-1} b$ is equivalent to $a x \preccurlyeq b$, so the image of $F$ is $\left\{x \in B_{\infty}^{+} ; a \preccurlyeq x \preccurlyeq b\right\}=C$. Finally, by construction, $F$ preserves both $\preccurlyeq$ and $<$.

For $r=1$, each $B_{r}$-class is a singleton, and Proposition 3.5 says nothing; similarly, if the main generator of $z$ is $\sigma_{m}$, there is only one $B_{r}$-class for $r>m$, and we gain no information. But, for $1<r \leqslant m$, and specially for $r=m$, Proposition 3.5 states that the chain $\operatorname{Div}(z)$ is obtained by concatenating $h_{r}(z)$ copies of sets of the form $\operatorname{Div}\left(z^{\prime}\right)$ with $z^{\prime}$ of index at most $r$. In particular, for $z=\Delta_{n}^{d}$, we have:

Corollary 3.6. For each $n$ and $r$ such that $r<n$, the chain $\left(\operatorname{Div}\left(\Delta_{n}^{d}\right),<\right)$ is obtained by concatenating $h_{r}\left(\Delta_{n}^{d}\right)$ intervals, each of which, when equipped with $\preccurlyeq$, is a translated copy of some initial sublattice of $\left(\operatorname{Div}\left(\Delta_{r}^{d}\right), \preccurlyeq\right)$.

The case of $\Delta_{3}^{2}$ and $\Delta_{4}$ are illustrated in Figure 7 and Figure 8.

3B. Extremal elements. The next step is to observe that extremal points in $B_{r}$ classes admit a simple characterization in terms of divisibility.

Proposition 3.7. Assume that $z$ is a positive braid.

(i) An element $x$ of $\operatorname{Div}(z)$ is the maximum of its $B_{r}$-class if and only if the relation $x \sigma_{i} \preccurlyeq z$ fails for $1 \leqslant i<r$. 
(ii) An element $x$ of $\operatorname{Div}(z)$ is the minimum of its $B_{r}$-class if and only if no $\sigma_{i}$ with $1 \leqslant i<r$ divides $x$ on the right.

Proof. (i) The condition is necessary: if $x \sigma_{i}$ lies in $\operatorname{Div}(z)$ for some $i$ with $i<r$, then $x \sigma_{i}$ lies in the same $B_{r}$-class as $x$, and it is larger both for $\preccurlyeq$ and $<$, so $x$ cannot be maximal in its $B_{r}$-class. Conversely, assume that $x$ is not maximal in its $B_{r}$-class. Then there exists $y$ satisfying $x<y$ and $y$ is $B_{r}$-equivalent to $x$. Now, by Proposition 3.5, the $1 \mathrm{~cm}$ of $x$ and $y$ is also $B_{r}$-equivalent to $x$, which means that there exists $y_{1}$ in $B_{r}^{+}$satisfying $\operatorname{lcm}(x, y)=x y_{1}$. Now $x<y$ implies

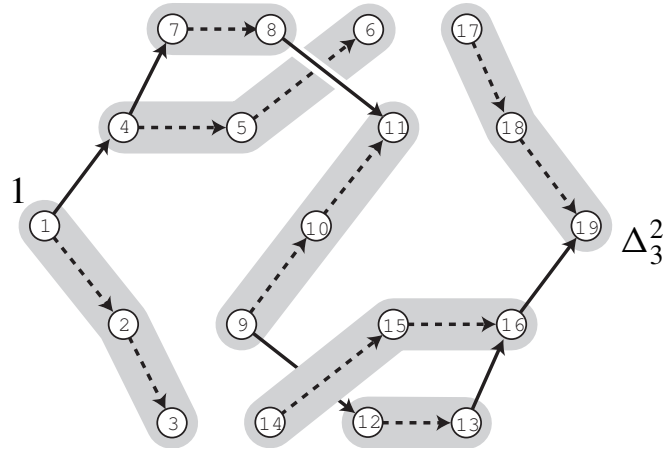

Figure 7. Decomposition of $\left(\operatorname{Div}\left(\Delta_{3}^{2}\right),<\right)$ into $B_{2}$-classes. The increasing enumeration of $\left(\operatorname{Div}\left(\Delta_{3}^{2}\right),<\right)$ is the concatenation of the increasing enumeration of the successive classes, separated by 2 -jumps (compare with Figure 4 ); in this case, $B_{2}$-classes are simply chains with respect to divisibility.



Figure 8. Decomposition of $\left(\operatorname{Div}\left(\Delta_{4}\right),<\right)$ into $B_{3}$-classes. The $\sigma_{3}$-arrows (thick) corresponding to 3 -jumps are not unique; in this case, all $B_{3}$-classes are isomorphic to the lattice $\left(\operatorname{Div}\left(\Delta_{3}\right),<, \preccurlyeq\right)$, that is, to the Cayley graph of $\Delta_{3}$. 
$y_{1} \neq 1$, so there must exist $i<m$ such that $\sigma_{i}$ is a left divisor of $y_{1}$. Then we have $x \sigma_{i} \preccurlyeq x y_{1} \preccurlyeq z$. Hence $x \sigma_{i} \preccurlyeq z$.

(ii) The argument is symmetric. If $x=y \sigma_{i}$ for some positive braid $y$ and $i<r$, then $y$ belongs to the $B_{r}$-class of $x$, and $x$ cannot be minimal in its $B_{r}$-class. Conversely, assume that $x$ is not minimal in its $B_{r}$-class. Then there exists $y$ satisfying $y<x$ and $y$ is $B_{r}$-equivalent to $x$. By Proposition 3.5 again, the gcd of $x$ and $y$ is also $B_{r}$ equivalent to $x$, which means that there exists $y_{0}$ in $B_{r}^{+} \operatorname{satisfying} \operatorname{gcd}(x, y) y_{0}=x$. As $y<x$ implies $y_{0} \neq 1$, there must exist $i<m$ such that $\sigma_{i}$ is a right divisor of $y_{0}$ and hence of $x$.

When we apply the previous criterion to the braids $\Delta_{n}^{d}$, we obtain:

Proposition 3.8. For $x$ in $\operatorname{Div}\left(\Delta_{n}^{d}\right)$ and $1 \leqslant r \leqslant n$, the following are equivalent.

(i) The element $x$ is <-maximal in its $B_{r}$-class.

(ii) The element $x \sigma_{i}$ belongs to $\operatorname{Div}\left(\Delta_{n}^{d}\right)$ for no $i<r$.

(iii) The d-th factor of $x$ is right divisible by $\Delta_{r}$.

(iv) The $(d+1)$-st factor of $x \Delta_{r}$ is $\Delta_{r}$.

Proof. The equivalence of (i) and (ii) is given by Proposition 3.7(i). It remains to establish the equivalence of (ii)-(iv). For $r=1$, (ii) is vacuously true, while (iii) and (iv) always hold. So the expected equivalences are true. We henceforth assume $r \geqslant 2$.

Let $x$ belong to $\operatorname{Div}\left(\Delta_{n}^{d}\right)$, and let $x_{d}$ be the $d$-th factor in the normal form of $x$. For $i<n$, saying that $x \sigma_{i}$ does not belong to $\operatorname{Div}\left(\Delta_{n}^{d}\right)$ means that the normal form of $x \sigma_{i}$ has length $d+1$. Hence, equivalently, that the normal form of $x_{d} \sigma_{i}$ has length 2. This occurs if and only if $\sigma_{i}$ is a right divisor of $x_{d}$. So, for $r \leqslant n$, (ii) is equivalent to $x_{d}$ being right divisible by all $\sigma_{i}$ 's with $1 \leqslant i<r$ and hence to $x_{d}$ being right divisible by the (left) lcm of these elements, which is $\Delta_{r}$.

Finally, (iii) and (iv) are equivalent. Indeed, if the $d$-th factor $x_{d}$ in the normal form of $x$ is divisible by $\Delta_{r}$ on the right, then $\left(x_{d}, \Delta_{r}\right)$ is a normal sequence as no $\sigma_{i}$ with $i<r$ from $\Delta_{r}$ may pass to $x_{d}$. Hence $\left(x_{1}, \ldots, x_{d}, \Delta_{r}\right)$ is a normal sequence and necessarily the normal form of $x \Delta_{r}$. Conversely, assume that the normal form of $x \Delta_{r}$ is $\left(x_{1}, \ldots, x_{d}, \Delta_{r}\right)$. The hypothesis that $\left(x_{d}, \Delta_{r}\right)$ is normal implies that $x_{d}$ is divisible on the right by each $\sigma_{i}$ with $i<r$. Hence is divisible on the right by $\Delta_{r}$. Now $\left(x_{1}, \ldots, x_{d}\right)$ is the normal form of $x$.

Observe that, for $r \geqslant 2$, an element of $\operatorname{Div}\left(\Delta_{n}^{d}\right)$ that is <-maximal in its $B_{r}$-class cannot belong to $\operatorname{Div}\left(\Delta_{n}^{d-1}\right)$, that is, it cannot have degree $d-1$ or less because the $d$-th factor of its normal form cannot be 1 . 
Similar conditions characterize the minimal elements of the $B_{r}$-classes. Because the normal form has a privileged orientation, the results are not entirely symmetric with those of Proposition 3.8

Proposition 3.9. For $x$ in $\operatorname{Div}\left(\Delta_{n}^{d}\right)$ and $1 \leqslant r \leqslant n$, the following are equivalent.

(i) The element $x$ is <-minimal in its $B_{r}$-class.

(ii) No $\sigma_{i}$ with $i<r$ is a right divisor of $x$.

(iii) The degrees of $x$ and $x \Delta_{r}$ are equal.

Proof. The equivalence of (i) and (ii) is given by Proposition 3.7(ii), and everything is obvious for $r=1$. So it remains to establish the equivalence of (ii) and (iii) when $r \geqslant 2$. Now, assume that (ii) holds and $x$ has degree $d$. The hypothesis that $\sigma_{i}$ is not a right divisor of $x$ implies that $x \sigma_{i}$ is a divisor of $\Delta_{n}^{d}$. As this holds for each $i<r$, the lcm of $x \sigma_{1}, \ldots, x \sigma_{r-1}$, namely $x \Delta_{r}$, also divides $\Delta_{n}^{d}$, which means that $x \Delta_{r}$ has degree (at most) $d$. So (ii) implies (iii).

Conversely, assume that $\sigma_{i}$ divides $x$ on the right. Then the degree of $x \sigma_{i}$ is strictly larger than that of $x$, and, a fortiori, the same is true for $x \Delta_{r}$.

3C. Determination of $\boldsymbol{h}_{\boldsymbol{r}}\left(\boldsymbol{\Delta}_{\boldsymbol{n}}^{\boldsymbol{d}}\right)$. A direct application of the previous results is a formula connecting the number $h_{r}\left(\Delta_{n}^{d}\right)$ of $B_{r}$-classes in $\operatorname{Div}\left(\Delta_{n}^{d}\right)$ with the number of braids whose normal form ends with some specific factor.

Definition 3.10. For $n, d \geqslant 1$ and for $s$ a simple $n$-braid, we denote by $b_{n, d}(s)$ the number of positive braids of degree at most $d$, that is, of divisors of $\Delta_{n}^{d}$, whose $d$-th factor is $s$.

Proposition 3.11. For $1 \leqslant r \leqslant n$, we have

$$
h_{r}\left(\Delta_{n}^{d}\right)=\sum_{s \text { right divisible by } \Delta_{r}} b_{n, d}(s)=b_{n, d+1}\left(\Delta_{r}\right) .
$$

In words, the number of $r$-jumps in $\left(\operatorname{Div}\left(\Delta_{n}^{d}\right),<\right)$ is the number of $n$-braids of degree at most $d$ whose $d$-th factor is right divisible by $\Delta_{r}$.

Proof. By Corollary 3.4, $h_{r}\left(\Delta_{n}^{d}\right)$ is the number of $B_{r}$-classes in $\operatorname{Div}\left(\Delta_{n}^{d}\right)$. Each class contains exactly one maximum element, and, by Proposition 3.8, its $d$-th factor is right divisible by $\Delta_{r}$. The first equality in (3-3) follows. The second one follows from the equivalence of (iii) and (iv) in Proposition 3.8 .

For $r=1$, as every simple braid is divisible by 1 on the right, Equation (3-3) reduces to

$$
h_{1}\left(\Delta_{n}^{d}\right)=\sum_{s} b_{n, d}(s)=b_{n, d+1}(1)
$$


a special case of the relation $h_{1}(z)=\# \operatorname{Div}(z)$ of Proposition 2.17. For $r=n$, because the only normal sequence of length $d$ that finishes with $\Delta_{n}$ is $\left(\Delta_{n}, \ldots, \Delta_{n}\right)$, Equation (3-3) reduces to

$$
h_{n}\left(\Delta_{n}^{d}\right)=1,
$$

already noted in Proposition 2.17. Finally, for $r=n-1$, we obtain using Proposition 2.19:

Corollary 3.12. For $n \geqslant 2$, we have

$$
c\left(\Delta_{n}^{d}\right)=h_{n-1}\left(\Delta_{n}^{d}\right)-1=\sum_{i=2}^{n} b_{n, d}\left(\sigma_{i} \sigma_{i+1} \ldots \sigma_{n-1} \Delta_{n-1}\right)=b_{n, d+1}\left(\Delta_{n-1}\right)-1 .
$$

Proof. The simple $n$-braids that are right divisible by $\Delta_{n-1}$ are the braids of the form $\sigma_{i} \sigma_{i+1} \ldots \sigma_{n-1}$ with $1 \leqslant i \leqslant n$. Indeed, it is clear that every such braid is simple and right divisible by $\Delta_{n-1}$. Conversely, the only possibility for $z \Delta_{n-1}$ to be simple is that $z$ moves the $n$-th strand to some position between 1 and $n$ without introducing any crossing between the remaining strands. Finally, $\sigma_{1} \sigma_{2} \ldots \sigma_{n-1} \Delta_{n-1}$ is $\Delta_{n}$, and, remembering that $b_{n, d}\left(\Delta_{n}\right)$ is 1 , we obtain the first equality.

3D. Computation of $\boldsymbol{b}_{n, d}(\boldsymbol{s})$. By Lemma 1.4, normal sequences are characterized by a local condition involving only pairs of consecutive elements. It follows that the set of all normal sequences is a rational set, that is, it can be recognized by a finite state automaton. Standard arguments then show that the numbers $b_{n, d}(s)$ obey a linear recurrence. Building on this observation, seemingly first used for braids in [Charney 1995], we can obtain explicit formulas for the parameters $c\left(\Delta_{n}^{d}\right)$ and $h_{r}\left(\Delta_{n}^{d}\right)$ for small values of $r, n$, or $d$. We shall not go into details here but refer to [Dehornoy 2007] where we established the formulas and, more generally, investigated the rich combinatorics underlying the normal form of braids.

In the sequel, we write $(M)_{x, y}$ for the $(x, y)$-entry of a matrix $M$. The general principle for computing the numbers $b_{n, d}(s)$ for some fixed $n$ is as follows:

Lemma 3.13. For $n \geqslant 1$, let $M_{n}$ be the square matrix with entries indexed by simple $n$-braids defined by

$$
\left(M_{n}\right)_{s, t}= \begin{cases}1 & \text { if }(s, t) \text { is normal }, \\ 0 & \text { otherwise. }\end{cases}
$$

Then, for every simple $t$ and $d \geqslant 1$, we have $b_{n, d}(t)=\left((1,1, \ldots, 1) M_{n}^{d-1}\right)_{t}$.

The proof is an easy induction on $d$.

Example 3.14. The matrix $M_{1}$ is (1), corresponding to $b_{1, d}(1)=1$. For $n=2$, using the enumeration $\left(1, \sigma_{1}\right)$ of simple 2-braids, we find $M_{2}=((1,0),(1,1))$, leading to $b_{2, d}(1)=d$ and $b_{2, d}\left(\sigma_{1}\right)=1$, giving $d+1$ braids of degree at most 
$d$. The first $d$ are the braids $\sigma_{1}^{e}$ with $e<d$ in which the $d$-th factor is 1 ; the last is $\sigma_{1}^{d}$, whose $d$-th factor is $\Delta_{2}$, that is, $\sigma_{1}$. For $n=3$, using the enumeration $\left(1, \sigma_{1}, \sigma_{2}, \sigma_{2} \sigma_{1}, \sigma_{1} \sigma_{2}, \Delta_{3}\right)$ of simple 3-braids, we obtain

$$
M_{3}=\left(\begin{array}{llllll}
1 & 0 & 0 & 0 & 0 & 0 \\
1 & 1 & 0 & 0 & 1 & 0 \\
1 & 0 & 1 & 1 & 0 & 0 \\
1 & 1 & 0 & 0 & 1 & 0 \\
1 & 0 & 1 & 1 & 0 & 0 \\
1 & 1 & 1 & 1 & 1 & 1
\end{array}\right),
$$

from which we can deduce $b_{3,3}(1)=19$ or $b_{3,4}\left(\sigma_{1}\right)=15$ using Lemma 3.13.

Using Proposition 3.11, we deduce:

Proposition 3.15. With $M_{n}$ as in Lemma 3.13, we have for $n \geqslant r \geqslant 1$ and $d \geqslant 1$

$$
\begin{aligned}
c\left(\Delta_{n}^{d}\right) & =\left((1,1, \ldots, 1) M_{n}^{d}\right)_{\Delta_{n-1}}-1, \\
h_{r}\left(\Delta_{n}^{d}\right) & =\left((1,1, \ldots, 1) M_{n}^{d}\right)_{\Delta_{r}} .
\end{aligned}
$$

Corollary 3.16. $\quad$ (i) For fixed $n, r$, the generating functions for the sequences $c\left(\Delta_{n}^{d}\right)$ and $h_{r}\left(\Delta_{n}^{d}\right)$ are rational.

(ii) For fixed $n, r$, the numbers $c\left(\Delta_{n}^{d}\right)$ and $h_{r}\left(\Delta_{n}^{d}\right)$ admit expressions of the form

$$
P_{1}(d) \rho_{1}^{d}+\cdots+P_{k}(d) \rho_{k}^{d} .
$$

where $\rho_{1}, \ldots, \rho_{k}$ are the nonzero eigenvalues of $M_{n}$ and $P_{1}, \ldots, P_{k}$ are polynomials with $\operatorname{deg}\left(P_{i}\right)$ of at most the multiplicity of $\rho_{i}$ in $M_{n}$.

Because the matrix $M_{n}$ is an $n ! \times n !$ matrix, completing the computation is not so easy, even for small values of $n$. Actually, it is shown in [Dehornoy 2007] how to replace $M_{n}$ with a smaller matrix $\bar{M}_{n}$ of size $p(n) \times p(n)$, where $p(n)$ is the number of partitions of $n$. The property is connected with classical results of Solomon [1976] about the descents of permutations. With such methods, one easily obtains the values listed in Table 1 .

Using the reduced matrices

$$
\bar{M}_{3}=\left(\begin{array}{lll}
1 & 0 & 0 \\
4 & 2 & 0 \\
1 & 1 & 1
\end{array}\right) \quad \text { and } \quad \bar{M}_{4}=\left(\begin{array}{ccccc}
1 & 0 & 0 & 0 & 0 \\
11 & 4 & 1 & 0 & 0 \\
5 & 3 & 2 & 1 & 0 \\
6 & 4 & 2 & 2 & 0 \\
1 & 1 & 1 & 1 & 1
\end{array}\right)
$$

we obtain the following explicit form for (3-4) involving the nonzero eigenvalues $(1,1,2)$ of $M_{3}$ and $(1,1,3 \pm \sqrt{6})$ of $M_{4}$ : 
Proposition 3.17. Let $\rho_{ \pm}=3 \pm \sqrt{6}$. Then, for $d \geqslant 1$, we have

$$
\begin{aligned}
& h_{1}\left(\Delta_{3}^{d}\right)=8 \cdot 2^{d}-3 d-7 \\
& h_{2}\left(\Delta_{3}^{d}\right)=c\left(\Delta_{3}^{d}\right)+1=2 \cdot 2^{d}-1 \\
& h_{1}\left(\Delta_{4}^{d}\right)=\sum_{ \pm} \frac{3}{20}(32 \pm 13 \sqrt{6}) \rho_{ \pm}^{d}-\frac{128}{5} \cdot 2^{d}+6 d+17 \\
& h_{2}\left(\Delta_{4}^{d}\right)=\sum_{ \pm} \frac{1}{20}(32 \pm 13 \sqrt{6}) \rho_{ \pm}^{d}-\frac{16}{5} \cdot 2^{d}+1 \\
& h_{3}\left(\Delta_{4}^{d}\right)=c\left(\Delta_{3}^{4}\right)+1=\sum_{ \pm} \frac{1}{20}(4 \pm \sqrt{6}) \rho_{ \pm}^{d}+\frac{8}{5} \cdot 2^{d}-1 .
\end{aligned}
$$

These formulas show each parameter grows exponentially in $d$, with estimate $\mathrm{O}\left(2^{d}\right)$ for $n=3$, and $\mathrm{O}\left((3+\sqrt{6})^{d}\right)$ for $n=4$. For practical purposes, it may be more convenient to resort to recursive formulas, for instance,

$$
\begin{aligned}
& h_{1}\left(\Delta_{3}^{d}\right)=2 h_{1}\left(\Delta_{3}^{d-1}\right)+3 d+1, \\
& h_{1}\left(\Delta_{4}^{d}\right)=6 h_{1}\left(\Delta_{4}^{d-1}\right)-3 h_{1}\left(\Delta_{4}^{d-2}\right)+32 \cdot 2^{d}-12 d-34,
\end{aligned}
$$

together with initial values $h_{1}\left(\Delta_{3}^{0}\right)=h_{1}\left(\Delta_{4}^{0}\right)=1, h_{1}\left(\Delta_{4}^{1}\right)=24\left(\right.$ or $\left.h_{1}\left(\Delta_{4}^{-1}\right)=0\right)$.

3E. Small values of $\boldsymbol{d}$. Another approach is to keep $d$ fixed and let $n$ vary. Once again, we only mention a few results, and refer the reader to [Dehornoy 2007] for the proofs and additional comments. For $d=1$, it is easy to determine all values:

Proposition 3.18 [Dehornoy 2007]. For $n \geqslant r \geqslant 1$, we have

$$
h_{r}\left(\Delta_{n}\right)=\frac{n !}{r !}
$$

For $d=2$, it is easier to complete the computation for $h_{n-r}\left(\Delta_{n}^{2}\right)$.

Proposition 3.19 [Dehornoy 2007]. For $n \geqslant r \geqslant 1$, we have

$$
h_{n-r}\left(\Delta_{n}^{2}\right)=r !(r+1)^{n}+\sum_{i=1}^{r} P_{i}(n) i^{n-r+i-1},
$$

for some polynomial $P_{i}$ of degree at most $r-i+1$. The values for $r=1,2$ are

$$
\begin{aligned}
& h_{n-1}\left(\Delta_{n}^{2}\right)=2^{n}-1, \\
& h_{n-2}\left(\Delta_{n}^{2}\right)=2 \cdot 3^{n}-(n+6) \cdot 2^{n-1}+1 .
\end{aligned}
$$


For $h_{r}\left(\Delta_{n}^{2}\right)$ itself, no general formula is known. We mention the case of $h_{1}\left(\Delta_{n}^{2}\right)$, which follows from results of Carlitz et al. [1976]:

Proposition 3.20 [Dehornoy 2007]. The numbers $h_{1}\left(\Delta_{n}^{2}\right)$ are determined by the induction

$$
h_{1}\left(\Delta_{0}^{2}\right)=1, \quad h_{1}\left(\Delta_{n}^{2}\right)=\sum_{i=0}^{n-1}(-1)^{n+i+1}\left(\begin{array}{l}
n \\
i
\end{array}\right)^{2} h_{1}\left(\Delta_{i}^{2}\right) .
$$

Their double exponential generating function is, with $J_{0}(x)$ is the Bessel function,

$$
\sum_{n=0}^{\infty} h_{1}\left(\Delta_{n}^{2}\right) \frac{z^{n}}{n !^{2}}=\left(\sum_{n=0}^{\infty}(-1)^{n} \frac{z^{n}}{n !^{2}}\right)^{-1}=\frac{1}{J_{0}(\sqrt{z})} .
$$

Finally, for $d=3$, the computation can be completed at least in the case $n-r=1$ :

Proposition 3.21 [Dehornoy 2007]. For $n \geqslant 1$, we have, with $e=\exp (1)$,

$$
h_{n-1}\left(\Delta_{n}^{3}\right)=\sum_{i=0}^{n-1} \frac{n !}{i !}=\lfloor n ! e\rfloor-1 .
$$

\begin{tabular}{crrrrrrr}
\hline$d$ & 0 & 1 & 2 & 3 & 4 & 5 & 6 \\
\hline$h_{1}\left(\Delta_{2}^{d}\right)$ & 1 & 2 & 3 & 4 & 5 & 6 & 7 \\
$h_{1}\left(\Delta_{3}^{d}\right)$ & 1 & 6 & 19 & 48 & 109 & 234 & 487 \\
$h_{2}\left(\Delta_{3}^{d}\right)$ & 1 & 3 & 7 & 15 & 31 & 63 & 127 \\
$h_{1}\left(\Delta_{4}^{d}\right)$ & 1 & 24 & 211 & 1,380 & 8,077 & 45,252 & 249,223 \\
$h_{2}\left(\Delta_{4}^{d}\right)$ & 1 & 12 & 83 & 492 & 2,765 & 15,240 & 83,399 \\
$h_{3}\left(\Delta_{4}^{d}\right)$ & 1 & 4 & 15 & 64 & 309 & 1,600 & 8,547 \\
$h_{1}\left(\Delta_{5}^{d}\right)$ & 1 & 120 & 3,651 & 79,140 & $1,548,701$ & $29,375,460$ & $551,997,751$ \\
$h_{2}\left(\Delta_{5}^{d}\right)$ & 1 & 60 & 1,501 & 30,540 & 585,811 & $11,044,080$ & $207,154,921$ \\
$h_{3}\left(\Delta_{5}^{d}\right)$ & 1 & 20 & 311 & 5,260 & 94,881 & $1,755,360$ & $32,741,851$ \\
$h_{4}\left(\Delta_{5}^{d}\right)$ & 1 & 5 & 31 & 325 & 4,931 & 86,565 & $1,590,231$ \\
$h_{1}\left(\Delta_{6}^{d}\right)$ & 1 & 720 & 90,921 & $7,952,040$ & $634,472,921$ & $49,477,263,360$ & $3,836,712,177,121$ \\
$h_{2}\left(\Delta_{6}^{d}\right)$ & 1 & 360 & 38,559 & $3,228,300$ & $254,718,389$ & $19,808,530,620$ & $1,535,016,069,499$ \\
$h_{3}\left(\Delta_{6}^{d}\right)$ & 1 & 120 & 8,727 & 649,260 & $49,654,757$ & $3,831,626,580$ & $296,361,570,667$ \\
$h_{4}\left(\Delta_{6}^{d}\right)$ & 1 & 30 & 1,075 & 61,620 & $4,387,195$ & $332,578,230$ & $25,612,893,355$ \\
$h_{5}\left(\Delta_{6}^{d}\right)$ & 1 & 6 & 63 & 1,956 & 116,423 & $8,448,606$ & $643,888,543$ \\
\hline
\end{tabular}

Table 1. First values of $h_{r}\left(\Delta_{n}^{d}\right)$ for $1 \leqslant r<n-$ the value is 1 for $r \geqslant n$. For instance, the number $h_{1}\left(\Delta_{3}^{2}\right)$ of 3-strand braids of degree at most 2 is 19 (see Example 2.16), while the maximal number $c\left(\Delta_{4}^{4}\right)$ of $\sigma_{3}$ 's in a $\sigma$-positive word drawn in $\Gamma\left(\Delta_{4}^{4}\right)-$ which is $h_{3}\left(\Delta_{4}^{4}\right)-1$, according to Proposition 2.19 - is 308 . 
Using Proposition 2.19, we deduce the following explicit values for $c\left(\Delta_{n}^{d}\right)$, that is, for the maximal number of occurrences of $\sigma_{n-1}$ in a $\sigma$-positive word drawn in the Cayley graph of $\Delta_{n}^{d}$ :

$$
c\left(\Delta_{n}\right)=n-1, \quad c\left(\Delta_{n}^{2}\right)=2^{n}-2, \quad c\left(\Delta_{n}^{3}\right)=\sum_{i=0}^{n-1} \frac{n !}{i !}-1=\lfloor n ! e\rfloor-2 .
$$

The formulas listed above show that a number of different induction schemes appear, suggesting that the combinatorics of normal sequences of braids is very rich.

\section{A complete description of $\left(\operatorname{Div}\left(\Delta_{3}^{d}\right),<\right)$}

Our ultimate goal is a complete description of each chain $\left(\operatorname{Div}\left(\Delta_{n}^{d}\right),<\right)$. Typically, this means that we are able to explicitly specify the increasing enumeration of its elements. The goal remains generally out of reach, but we can show how the process can be completed when $n=3$. The counting formulas of Section 3 play a key role in the construction, and, in particular, the Pascal's triangle of Figure 9 connects directly with the $2^{d}$ factor in the inductive formulas of Proposition 3.17. As an application, we deduce a new proof of Property $\mathrm{C}$ and of the well-ordering property and hence a complete reconstruction of the braid ordering when $n=3$.

The general principle is to make the decomposition of Corollary 3.6 explicit. The latter shows that, for all $n$ and $d$, the chain $\left(\operatorname{Div}\left(\Delta_{n}^{d}\right),<\right)$ can be decomposed into $c\left(\Delta_{n}^{d}\right)$ subintervals each of which copies some fragment of $\left(\operatorname{Div}\left(\Delta_{n-1}^{d}\right),<\right)$. Moreover, the approach of Section 3 suggests an induction on $d$ as well. We are led to seek a recursion for $\left(\operatorname{Div}\left(\Delta_{n}^{d}\right),<\right)$ in $\left(\operatorname{Div}\left(\Delta_{n-1}^{d}\right),<\right)$ and $\left(\operatorname{Div}\left(\Delta_{n}^{d-1}\right),<\right)$; here this means expressing $\left(\operatorname{Div}\left(\Delta_{3}^{d}\right),<\right)$ in $\left(\operatorname{Div}\left(\Delta_{2}^{d}\right),<\right)$ and $\left(\operatorname{Div}\left(\Delta_{3}^{d-1}\right),<\right)$.

4A. The braids $\boldsymbol{\theta}_{\boldsymbol{n}, \boldsymbol{p}}$. The subsequent construction will appeal to a double series $\theta_{n, p}$ of braids, and we begin with a few preliminary properties.

Definition 4.1. For $n \geqslant 2$, let $\sigma_{n, 1}$ and $\sigma_{1, n}$ denote the braid words $\sigma_{n-1} \sigma_{n-2} \ldots \sigma_{1}$ and $\sigma_{1} \sigma_{2} \ldots \sigma_{n-1}$. For $p \geqslant 0$, we define $\widetilde{\theta}_{n, p}$ as (the braid represented by) the length $p$ prefix of the right-infinite word $\left(\sigma_{n, 1} \sigma_{1, n}\right)^{\infty}$, and let $\theta_{n, p}$ be (the braid represented by) the length $p$ suffix of the left-infinite word ${ }^{\infty}\left(\sigma_{n, 1} \sigma_{1, n}\right)$.

For instance, we find $\theta_{3,0}=1, \theta_{3,1}=\mathrm{b}, \theta_{3,2}=\mathrm{ab}, \ldots, \theta_{3,4}=\mathrm{baab}, \ldots, \theta_{3,7}=$ aabbaab, etc. Similarly, we have $\theta_{4,6}=$ cbaabc and, more generally, $\theta_{n, 2 n-2}=$ $\widetilde{\theta}_{n, 2 n-2}=\sigma_{n, 1} \sigma_{1, n}$. Note that, as words, $\theta_{n, p}$ is the reverse of $\widetilde{\theta}_{n, p}$.

Lemma 4.2. For $n \geqslant 2$ and $p, q \geqslant 0$ satisfying $p+q=d(n-1)$, we have

$$
\theta_{n, p} \Delta_{n-1}^{d} \tilde{\theta}_{n, q}=\Delta_{n}^{d}
$$


Proof. We first prove using induction on $d$ the relation

$$
\theta_{n, d(n-1)} \Delta_{n-1}^{d}=\Delta_{n}^{d},
$$

that is, (4-1) with $q=0$. For $d=0$, (4-2) reduces to $1=1$. Assume $d \geqslant 1$. By definition, $\theta_{n, d(n-1)}$ is $\sigma_{n, 1} \theta_{n,(d-1)(n-1)}$ for $d$ odd and is $\sigma_{1, n} \theta_{n,(d-1)(n-1)}$ for $d$ even. In either case, we can write

$$
\theta_{n, d(n-1)}=\phi_{n}^{d-1}\left(\sigma_{1, n}\right) \theta_{n,(d-1)(n-1)},
$$

where we recall $\phi_{n}$ denotes the flip automorphism of $B_{n}$ that exchanges $\sigma_{i}$ and $\sigma_{n-i}$. Using the induction hypothesis and (1-2), we find

$$
\begin{aligned}
\theta_{n, d(n-1)} \Delta_{n-1}^{d} & =\phi_{n}^{d-1}\left(\sigma_{1, n}\right) \theta_{n,(d-1)(n-1)} \Delta_{n-1}^{d-1} \Delta_{n-1} \\
& =\phi_{n}^{d-1}\left(\sigma_{1, n}\right) \Delta_{n}^{d-1} \Delta_{n-1}=\Delta_{n}^{d-1} \sigma_{1, n} \Delta_{n-1}=\Delta_{n}^{d-1} \Delta_{n}=\Delta_{n}^{d} .
\end{aligned}
$$

We return to the general case of (4-1). For $d$ even, we have $\theta_{n, d(n-1)}=\widetilde{\theta}_{n, d(n-1)}$ and hence $\widetilde{\theta}_{n, q} \theta_{n, p}=\theta_{n, d(n-1)}$. If $d$ is odd, we have $\theta_{n, d(n-1)}=\phi_{n}\left(\widetilde{\theta}_{n, d(n-1)}\right)$, which implies $\phi_{n}\left(\widetilde{\theta}_{n, q}\right) \theta_{n, p}=\theta_{n, d(n-1)}$. So $\phi_{n}^{d}\left(\widetilde{\theta}_{n, q}\right) \theta_{n, p}=\theta_{n, d(n-1)}$ holds in both cases. Now, using (4-2), we find

$$
\phi_{n}\left(\widetilde{\theta}_{n, q}\right) \theta_{n, p} \Delta_{n-1}^{d} \widetilde{\theta}_{n, q}=\theta_{n, d(n-1)} \Delta_{n-1}^{d} \tilde{\theta}_{n, q}=\Delta_{n}^{d} \widetilde{\theta}_{n, q}=\phi_{n}\left(\tilde{\theta}_{n, q}\right) \Delta_{n}^{d},
$$

from which we deduce (4-1) by cancelling $\phi_{n}\left(\widetilde{\theta}_{n, q}\right)$ on the left.

Lemma 4.3. For $1 \leqslant i \leqslant n-2$ we have

$$
\theta_{n, d(n-1)} \sigma_{i}=\sigma_{i+e} \theta_{n, d(n-1)}
$$

with $e=0$ if $d$ is even and $e=1$ if $d$ is odd.

Proof. For $1 \leqslant i \leqslant n-2$, we have $\sigma_{1, n} \sigma_{i}=\sigma_{i+1} \sigma_{1, n}$ and $\sigma_{n, 1} \sigma_{i+1}=\sigma_{i} \sigma_{n, 1}$, as an easy induction shows. This implies $\sigma_{n, 1} \sigma_{1, n} \sigma_{i}=\sigma_{i} \sigma_{n, 1} \sigma_{1, n}$ and therefore $\left(\sigma_{n, 1} \sigma_{1, n}\right)^{d} \sigma_{i}=\sigma_{i}\left(\sigma_{n, 1} \sigma_{1, n}\right)^{d}$, that is, $\theta_{n, 2 d(n-1)} \sigma_{i}=\sigma_{i} \theta_{n, 2 d(n-1)}$ for every $d$. On the other hand, we have $\theta_{n,(2 d+1)(n-1)}=\sigma_{1, n} \theta_{n, 2 d(n-1)}$ and hence

$$
\theta_{n,(2 d+1)(n-1)} \sigma_{i}=\sigma_{1, n} \sigma_{i} \theta_{n, 2 d(n-1)}=\sigma_{i+1} \sigma_{1, n} \theta_{n, 2 d(n-1)}=\sigma_{i+1} \theta_{n,(2 d+1)(n-1)},
$$

as was expected.

4B. A Pascal triangle. We shall now construct for every $d$ a sequence of positive braids $S_{3}^{d}$ that will be the increasing enumeration of $\left(\operatorname{Div}\left(\Delta_{3}^{d}\right),<\right)$. The construction relies on an induction similar to Pascal's triangle. To make it easily understandable, we start with the (trivial) cases $n=1$ and $n=2$.

Because $B_{1}$ is the trivial group, for every $d, 1$ is the only element of degree at most $d$, and we can state: 
Proposition 4.4. Define $S_{1}^{d}$ for $d \geqslant 0$ by

$$
S_{1}^{d}=(1) \text {. }
$$

Then $S_{1}^{d}$ is the increasing enumeration of $\operatorname{Div}\left(\Delta_{1}^{d}\right)$.

The group $B_{2}$ is the rank 1 free group generated by $\sigma_{1}$. The braid $\Delta_{2}$ is just $\sigma_{1}$, and the braids of degree at most $d$, that is, the divisors of $\Delta_{2}^{d}$, consist of the $d+1$ braids $1, \sigma_{1}, \ldots, \sigma_{1}^{d}$. On the other hand, we have $\sigma_{1,2}=\sigma_{2,1}=\sigma_{1}$, and $\theta_{1, i}=\sigma_{1}^{i}$ for every $i$.

Notation 4.5. If $S_{1}, S_{2}$ are sequences (of braids), we denote by $S_{1}+S_{2}$ the (ordered) concatenation of $S_{1}$ and $S_{2}$. If $S$ is a sequence of braids and $x$ is a braid, we denote by $x S$ the translated sequence obtained by multiplying each entry in $S$ by $x$ on the left.

In these terms, the sequence $\left(1, \sigma_{1}, \ldots, \sigma_{1}^{d}\right)$ can be expressed as a sum of sequences $\theta_{2,0}(1)+\theta_{2,1}(1)+\cdots+\theta_{2, d}(1)$. Hence:

Proposition 4.6. Define $S_{2}^{d}$ for $d \geqslant 0$ by

$$
S_{2}^{d}=\theta_{2,0} S_{1}^{d}+\theta_{2,1} S_{1}^{d}+\cdots+\theta_{2, d} S_{1}^{d} .
$$

Then $S_{2}^{d}$ is the increasing enumeration of $\operatorname{Div}\left(\Delta_{2}^{d}\right)$.

We repeat the process for $n=3$, introducing a sequence $S_{3}^{d}$ by a definition similar to (4-4) that involves $S_{2}^{d}$ and $S_{3}^{d-1}$. The result we shall prove is:

Proposition 4.7. Let $S_{3}^{d}$ be defined for $d \geqslant 0$ by

$$
S_{3}^{d}=\theta_{3,0} S_{2}^{d}+S_{3}^{d, 1}+\theta_{3,1} S_{2}^{d}+\cdots+\theta_{3,2 d-1} S_{2}^{d}+S_{3}^{d, 2 d}+\theta_{3,2 d} S_{2}^{d},
$$

where $S_{3}^{d, 1}, \cdots, S_{3}^{d, 2 d}$ are defined by $S_{3}^{d, 1}=S_{3}^{d, 2 d}=\varnothing$ and, for $2 \leqslant p \leqslant 2 d-1$,

$$
S_{3}^{d, p}=\left\{\begin{array}{ccc}
\sigma_{1}\left(S_{3}^{d-1, p-1}+\theta_{3, p-1} S_{2}^{d-1}+S_{3}^{d-1, p}\right) & \text { for } p=0 & (\bmod 4), \\
\sigma_{2} \sigma_{1}\left(S_{3}^{d-1, p-2}+\theta_{3, p-1} S_{2}^{d-1}+S_{3}^{d-1, p-1}\right) & \text { for } p=1 & (\bmod 4), \\
\sigma_{2}\left(S_{3}^{d-1, p-1}+\theta_{3, p-1} S_{2}^{d-1}+S_{3}^{d-1, p}\right) & \text { for } p=2 & (\bmod 4), \\
\sigma_{1} \sigma_{2}\left(S_{3}^{d-1, p-2}+\theta_{3, p-1} S_{2}^{d-1}+S_{3}^{d-1, p-1}\right) & \text { for } p=3 & (\bmod 4) .
\end{array}\right.
$$

Then $S_{3}^{d}$ is the increasing enumeration of $\operatorname{Div}\left(\Delta_{3}^{d}\right)$.

The general scheme is illustrated in Figure 9. The sequence $S_{3}^{d}$ is constructed by starting with $2 d+1$ copies of $S_{2}^{d}$ translated by $\theta_{3,0}, \ldots, \theta_{3,2 d}$ and inserting (translated copies of) fragments of the previous sequence $S_{3}^{d-1}$.

Example 4.8. The difference between the definition of $S_{3}^{d}$ in (4-5) and that of $S_{2}^{d}$ in (4-4) is the insertion of the additional factors $S_{3}^{d, p}$ between the consecutive terms 


$$
\begin{aligned}
& \theta_{3,0} S_{2}^{0} \\
& \theta_{3,0} S_{2}^{1} \underbrace{\left(S_{3}^{1,1}\right) \theta_{3,1} S_{2}^{1}\left(S_{3}^{1,2}\right)}_{\sigma_{2} \vee} \theta_{\sigma_{1,2} \sigma_{2}} S_{2}^{1}
\end{aligned}
$$

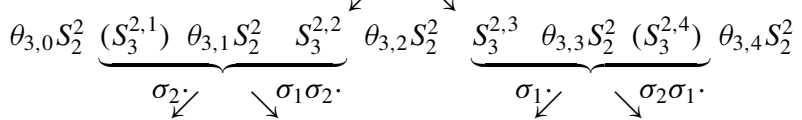

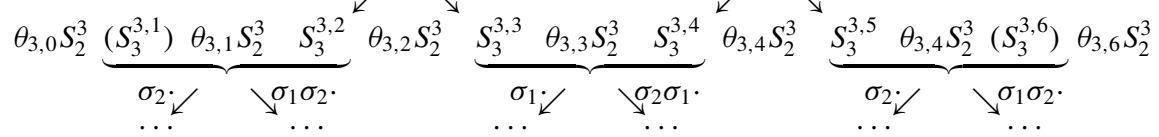

Figure 9. The inductive construction of $S_{3}^{d}$ as a Pascal triangle: the subsequence $S_{3}^{d, p}$ is obtained by translating and concatenating the previous subsequences $S_{3}^{d-1, p-1}$ and $S_{3}^{d-1, p}$, or $S_{3}^{d-1, p-2}$ and $S_{3}^{d-1, p-1}$, depending on the parity of $p$. The bracketed sequences are empty; if we remove the subsequences $\theta_{3, q} S_{2}^{d}$, we have the Pascal triangle.

$\theta_{3, q} S_{2}^{d}$. Because $S_{3}^{d, 1}$ and $S_{3}^{d, 2 d}$ are empty, the difference occurs for $d \geqslant 2$ only. The first values are:

$$
\begin{aligned}
S_{3}^{0}= & \theta_{3,0} S_{2}^{0}=(1), \\
S_{3}^{1}= & \theta_{3,0} S_{2}^{1}+S_{3}^{1,1}+\theta_{3,1} S_{2}^{1}+S_{3}^{1,2}+\theta_{3,2} \\
= & (1, \mathrm{a})+\varnothing+\mathrm{b}(1, \mathrm{a})+\varnothing+\mathrm{ab}(1, \mathrm{a})=(1, \mathrm{a}, \mathrm{b}, \mathrm{ba}, \mathrm{ab}, \mathrm{aba}), \\
S_{3}^{2}= & \theta_{3,0} S_{2}^{2}+S_{3}^{2,1}+\theta_{3,1} S_{2}^{2}+S_{3}^{2,2}+\theta_{3,2} S_{2}^{2}+S_{3}^{2,3}+\theta_{3,3} S_{2}^{2}+S_{3}^{2,4}+\theta_{3,4} S_{2}^{2} \\
= & (1, \mathrm{a}, \mathrm{aa})+\varnothing+\mathrm{b}(1, \mathrm{a}, \mathrm{aa})+\mathrm{b}(\mathrm{b}, \mathrm{ba})+\mathrm{ab}(1, \mathrm{a}, \mathrm{aa}) \\
& \quad+\mathrm{ab}(\mathrm{b}, \mathrm{ba})+\mathrm{aab}(1, \mathrm{a}, \mathrm{aa})+\varnothing+\mathrm{baab}(1, \mathrm{a}, \mathrm{aa}) \\
= & (1, \mathrm{a}, \mathrm{aa}, \mathrm{b}, \mathrm{ba}, \mathrm{baa}, \mathrm{bb}, \mathrm{bba}, \mathrm{ab}, \mathrm{aba}, \mathrm{abaa}, \mathrm{abb}, \mathrm{abba}, \text { aab }, \\
& \quad \text { aaba, aabaa, baab, baaba, baabaa }) .
\end{aligned}
$$

It is easy to check directly that the sequence $S_{3}^{d}$ provides the increasing enumeration of $\operatorname{Div}\left(\Delta_{3}^{d}\right)$ for $d=0,1,2$.

The proof of Proposition 4.7 will be split into several pieces, each of which is established using an induction on the degree $d$.

Lemma 4.9. All entries in $S_{3}^{d}$ are divisors of $\Delta_{3}^{d}$.

Proof. The result is true for $d=0$. Assume $d \geqslant 1$. By construction, each entry in $S_{3}^{d}$ either is of the form $\theta_{3, q} \sigma_{1}^{e}$ with $0 \leqslant q \leqslant 2 d$ and $0 \leqslant e \leqslant d$ or belongs to some subsequence $S_{3}^{d, p}$ with $2 \leqslant p \leqslant 2 d-1$. In the first case, $\theta_{3, q} \sigma_{1}^{e}$ is a right divisor of $\theta_{3,2 d} \sigma_{1}^{e}$, which itself is a left divisor of $\theta_{3,2 d} \sigma_{1}^{d}$. By Equation (4-1), the 
latter is $\Delta_{3}^{d}$. Hence each $\theta_{3, q} \sigma_{1}^{e}$ is a divisor of $\Delta_{3}^{d}$. As for the entries coming from some subsequence $S_{3}^{d, p}$, by definition they are of the form $x y$ with $x$ one of $\sigma_{2}, \sigma_{1} \sigma_{2}, \sigma_{1}, \sigma_{2} \sigma_{1}$ and $y$ an entry in $S_{3}^{d-1}$. Then $x$ is a divisor of $\Delta_{3}$, while, by the induction hypothesis, $y$ is a divisor of $\Delta_{3}^{d-1}$. Thus $x y$ is a divisor of $\Delta_{3}^{d}$.

Lemma 4.10. The length of the sequence $S_{3}^{d}$ equals the cardinality of $\operatorname{Div}\left(\Delta_{3}^{d}\right)$.

Proof. Let $\ell_{d}$ denote the length of $S_{3}^{d}$. Computing $\ell_{d}$ by recursion is not very difficult but also unnecessary. Indeed, we saw in Section 3 that the cardinality $h_{1}\left(\Delta_{3}^{d}\right)$ of $\operatorname{Div}\left(\Delta_{3}^{d}\right)$ obeys the inductive rule (3-5). So it will be enough to check that $\ell_{d}$ satisfies the relation

$$
\ell_{d}=2 \ell_{d-1}+3 d+1
$$

and starts from the initial $\ell_{1}=6$ (or $\ell_{0}=1$ ). The latter point was checked in Example 4.8.

Figure 9 shows that most entries in $S_{3}^{d-1}$ generate two entries in $S_{3}^{d}$. More precisely, each entry of $S_{3}^{d-1}$ not belonging to a factor of the form $\theta_{3,2 q} S_{2}^{d-1}$ generates two entries in $S_{3}^{d}$, and, conversely, each entry in $S_{3}^{d}$ not belonging to a factor $\theta_{3, q} S_{2}^{d}$ comes from such an entry in $S_{3}^{d-1}$. The $d$ factors $\theta_{3,2 q} S_{2}^{d-1}$ in $S_{3}^{d-1}$ each have length $d$, and the $2 d+1$ factors $\theta_{3,2 q} S_{2}^{d}$ in $S_{3}^{d}$ each have length $d+1$. So we obtain

$$
\ell_{d}-(2 d+1)(d+1)=2\left(\ell_{d-1}-d^{2}\right),
$$

which gives Equation (4-6).

At this point, we cannot (yet) conclude that each divisor of $\Delta_{3}^{d}$ occurs exactly once in $S_{3}^{d}$, as there could be some repetitions.

4C. A quotient sequence for $S_{3}^{d}$. Our next aim is to show that $S_{3}^{d}$ is <-increasing. To this end, we shall explicitly determine the quotient of adjacent entries in $S_{3}^{d}$, that is, we shall specify a quotient sequence for $S_{3}^{d}$ in the sense of Definition 2.22.

We begin by determining the first and the last entries of the sequence $S_{3}^{d, p}$. For $S$ a nonempty sequence, we denote by $(S)_{1}$ and $(S)_{\infty}$ the first and last entry in $S$.

Lemma 4.11. For $1<p<2 d$, we have

$$
\left(S_{3}^{d, p}\right)_{1}=\theta_{3, p-1} \sigma_{2} \quad \text { and } \quad\left(S_{3}^{d, p}\right)_{\infty} \sigma_{2}=\theta_{3, p} \sigma_{1}^{d} .
$$

Proof. The result is vacuously true for $d=0,1$. Assume $d \geqslant 2$ with $p=0(\bmod 4)$. Using the definition, the induction hypothesis, and (4-3), we find

$$
\begin{aligned}
& \left(S_{3}^{d, p}\right)_{1}=\sigma_{1}\left(S_{3}^{d-1, p-1}\right)_{1}=\sigma_{1} \theta_{3, p-2} \sigma_{2}=\theta_{3, p-1} \sigma_{2}, \\
& \left(S_{3}^{d, p}\right)_{\infty} \sigma_{2}=\sigma_{1}\left(S_{3}^{d-1, p}\right)_{\infty} \sigma_{2}=\sigma_{1} \theta_{3, p} \sigma_{1}^{d-1}=\theta_{3, p} \sigma_{1}^{d} .
\end{aligned}
$$


Similarly, for $p=1(\bmod 4)$, we have

$$
\begin{aligned}
& \left(S_{3}^{d, p}\right)_{1}=\sigma_{2} \sigma_{1}\left(S_{3}^{d-1, p-2}\right)_{1}=\sigma_{2} \sigma_{1} \theta_{3, p-3} \sigma_{2}=\theta_{3, p-1} \sigma_{2}, \\
& \left(S_{3}^{d, p}\right)_{\infty} \sigma_{2}=\sigma_{2} \sigma_{1}\left(S_{3}^{d-1, p-1}\right)_{\infty} \sigma_{2}=\sigma_{2} \sigma_{1} \theta_{3, p-1} \sigma_{1}^{d-1}=\sigma_{2} \theta_{3, p-1} \sigma_{1}^{d}=\theta_{3, p} \sigma_{1}^{d} .
\end{aligned}
$$

Then, for $p=2(\bmod 4)$, we have

$$
\begin{aligned}
& \left(S_{3}^{d, p}\right)_{1}=\sigma_{2}\left(S_{3}^{d-1, p-1}\right)_{1}=\sigma_{2} \theta_{3, p-2} \sigma_{2}=\theta_{3, p-1} \sigma_{2}, \\
& \left(S_{3}^{d, p}\right)_{\infty} \sigma_{2}=\sigma_{2}\left(S_{3}^{d-1, p}\right)_{\infty} \sigma_{2}=\sigma_{2} \theta_{3, p} \sigma_{1}^{d-1}=\theta_{3, p} \sigma_{1}^{d} .
\end{aligned}
$$

Finally, for $p=3(\bmod 4)$, we find

$$
\begin{gathered}
\left(S_{3}^{d, p}\right)_{1}=\sigma_{1} \sigma_{2}\left(S_{3}^{d-1, p-2}\right)_{1}=\sigma_{1} \sigma_{2} \theta_{3, p-3} \sigma_{2}=\theta_{3, p-1} \sigma_{2} \\
\left(S_{3}^{d, p}\right)_{\infty} \sigma_{2}=\sigma_{1} \sigma_{2}\left(S_{3}^{d-1, p-1}\right)_{\infty} \sigma_{2}=\sigma_{1} \sigma_{2} \theta_{3, p-1} \sigma_{1}^{d-1}=\sigma_{1} \sigma_{2} \sigma_{1} \sigma_{2} \theta_{3, p-3} \sigma_{1}^{d-1} \\
=\sigma_{1} \sigma_{1} \sigma_{2} \sigma_{1} \theta_{3, p-3} \sigma_{1}^{d-1}=\sigma_{1} \sigma_{1} \sigma_{2} \theta_{3, p-3} \sigma_{1}^{d}=\theta_{3, p} \sigma_{1}^{d}
\end{gathered}
$$

We shall now construct an explicit quotient sequence for $S_{3}^{d}$, that is, a sequence of braid words representing the quotients of the consecutive entries of $S_{3}^{d}$. Before doing it for $S_{3}^{d}$, let us consider the (trivial) cases of $S_{1}^{d}$ and $S_{2}^{d}$. As $S_{1}^{d}$ consists of one single entry, it vacuously admits the empty sequence as a quotient sequence. As for $S_{2}^{d}$, we can state:

Lemma 4.12. For $d \geqslant 0$, let $\boldsymbol{w}_{1}^{d}$ be the empty sequence, and let $\boldsymbol{w}_{2}^{d}$ be defined by

$$
\boldsymbol{w}_{2}^{d}=\boldsymbol{w}_{1}^{d}+\left(\sigma_{1}\right)+\boldsymbol{w}_{1}^{d}+\cdots+\boldsymbol{w}_{1}^{d}+\left(\sigma_{1}\right)+\boldsymbol{w}_{1}^{d},
$$

$d$ times $\left(\sigma_{1}\right)$. Then $\boldsymbol{w}_{2}^{d}$ is a quotient sequence for $S_{2}^{d}$.

In a similar way, we shall prove:

Proposition 4.13. Let $\boldsymbol{w}_{3}^{d}$ be the sequence defined by $\boldsymbol{w}_{3}^{0}=\varnothing$ and

$$
\begin{aligned}
\boldsymbol{w}_{3}^{d}=\boldsymbol{w}_{2}^{d}+\left(\sigma_{1}^{-d} \sigma_{2}\right) & +\boldsymbol{w}_{2}^{d}+\left(\sigma_{1}^{-d} \sigma_{2}\right)+\boldsymbol{w}_{3}^{d, 2}+\left(\sigma_{2} \sigma_{1}^{-d}\right) \\
& +\boldsymbol{w}_{2}^{d}+\left(\sigma_{1}^{-d} \sigma_{2}\right)+\boldsymbol{w}_{3}^{d, 3}+\left(\sigma_{2} \sigma_{1}^{-d}\right)+\cdots \\
& +\boldsymbol{w}_{2}^{d}+\left(\sigma_{1}^{-d} \sigma_{2}\right)+\boldsymbol{w}_{3}^{d, 2 d-1}+\left(\sigma_{2} \sigma_{1}^{-d}\right) \\
& +\boldsymbol{w}_{2}^{d}+\left(\sigma_{2} \sigma_{1}^{-d}\right)+\boldsymbol{w}_{2}^{d},
\end{aligned}
$$

with

$$
\begin{aligned}
& \boldsymbol{w}_{3}^{d, 2}=\boldsymbol{w}_{3}^{d, 3}=\boldsymbol{w}_{2}^{d-1}+\left(\sigma_{2} \sigma_{1}^{-d+1}\right)+\boldsymbol{w}_{3}^{d-1,2}, \\
& \boldsymbol{w}_{3}^{d, 2 d-2}=\boldsymbol{w}_{3}^{d, 2 d-1}=\boldsymbol{w}_{3}^{d-1,2 d-3}+\left(\sigma_{1}^{-d+1} \sigma_{2}\right)+\boldsymbol{w}_{2}^{d-1}, \\
& \boldsymbol{w}_{3}^{d, 2 p}=\boldsymbol{w}_{3}^{d, 2 p+1}=\boldsymbol{w}_{3}^{d-1,2 p-1}+\left(\sigma_{1}^{-d+1} \sigma_{2}\right)+\boldsymbol{w}_{2}^{d-1}+\left(\sigma_{2} \sigma_{1}^{-d+1}\right)+\boldsymbol{w}_{3}^{d-1,2 p},
\end{aligned}
$$

for $4 \leqslant 2 p \leqslant 2 d-4$. Then $\boldsymbol{w}_{3}^{d}$ is a quotient sequence for $S_{3}^{d}$. 
Example 4.14. We find $\boldsymbol{w}_{3}^{1}=\boldsymbol{w}_{2}^{1}+(\mathrm{Ab})+\boldsymbol{w}_{2}^{1}+(\mathrm{bA})+\boldsymbol{w}_{2}^{1}=(\mathrm{a}, \mathrm{Ab}, \mathrm{a}, \mathrm{bA}, \mathrm{a})$, and

$$
\begin{aligned}
\boldsymbol{w}_{3}^{2}=\boldsymbol{w}_{2}^{2}+(\mathrm{AAb}) & +\boldsymbol{w}_{2}^{2}+(\mathrm{AAb})+\boldsymbol{w}_{3}^{2,2}+(\mathrm{bAA}) \\
& +\boldsymbol{w}_{2}^{2}+(\mathrm{AAb})+\boldsymbol{w}_{3}^{2,3}+(\mathrm{bAA})+\boldsymbol{w}_{2}^{2}+(\mathrm{bAA})+\boldsymbol{w}_{2}^{2}
\end{aligned}
$$

with $\boldsymbol{w}_{3}^{2,2}=\boldsymbol{w}_{3}^{2,3}=\boldsymbol{w}_{2}^{1}=(\mathrm{a})$, whence

$$
\boldsymbol{w}_{3}^{2}=(\mathrm{a}, \mathrm{a}, \mathrm{AAb}, \mathrm{a}, \mathrm{a}, \mathrm{AAb}, \mathrm{a}, \mathrm{bAA}, \mathrm{a}, \mathrm{a}, \mathrm{AAb}, \mathrm{a}, \mathrm{bAA}, \mathrm{a}, \mathrm{a}, \mathrm{bAA}, \mathrm{a}, \mathrm{a}) .
$$

Proof of Proposition 4.13. We prove using induction on $d$ that $\boldsymbol{w}_{3}^{d}$ is a quotient sequence for $S_{3}^{d}$ with the $4 d-2$ terms in (4-7) corresponding to the $4 d-1$ nonempty terms in (4-5). In particular, for $2 \leqslant p \leqslant 2 d-1$, the subsequence $w_{3}^{d, p}$ is a quotient sequence for $S_{3}^{d, p}$. The result is vacuously true for $d=0$. Assume $d \geqslant 1$. By definition, the sequence $S_{3}^{d}$ consists of the concatenation of the $2 d+1$ sequences $\theta_{3,0} S_{2}^{d}, \cdots, \theta_{3,2 d} S_{2}^{d}$, in which the $2 d-2$ sequences $S_{3}^{d, 2}, \ldots, S_{3}^{d, 2 d-1}$ are inserted. We shall consider these subsequences separately and then consider the transitions between consecutive subsequences.

First, since $\boldsymbol{w}_{2}^{d}$ is a quotient sequence for $S_{2}^{d}$, it is a quotient sequence for every sequence $\theta_{3, q} S_{2}^{d}$ as well, because, by definition, the quotients we consider are invariant under left translation. Then, by construction, each subsequence $S_{3}^{d, 2 p}$ or $S_{3}^{d, 2 p+1}$ appearing in $S_{3}^{d}$ is obtained by translating some subsequence $S$ of $S_{3}^{d-1}$, namely

$$
S=S_{3}^{d-1,2 p-1}+\theta_{3, q-1} S_{2}^{d-1}+S_{3}^{d-1,2 p} .
$$

By the induction hypothesis, the sequence

$$
\boldsymbol{w}_{3}^{d-1,2 p-1}+\left(\sigma_{1}^{-d+1} \sigma_{2}\right)+\boldsymbol{w}_{2}^{d-1}+\left(\sigma_{2} \sigma_{1}^{-d+1}\right)+\boldsymbol{w}_{3}^{d-1,2 p},
$$

which by definition is precisely $\boldsymbol{w}_{3}^{d, 2 p}$ and $\boldsymbol{w}_{3}^{d, 2 p+1}$, is a quotient sequence for $S$. The property remains true in the special cases $p=1$ and $p=d$, which correspond respectively to removing the initial term $S_{3}^{d-1,2 p-1}$ and the final term $S_{3}^{d-1,2 p}$. Then $\boldsymbol{w}_{3}^{d, 2 p}$ and $\boldsymbol{w}_{3}^{d, 2 p+1}$ are also quotient sequences for any sequence obtained from $S$ by a left translation, and, in particular, for $S_{3}^{d, 2 p}$ and $S_{3}^{d, 2 p+1}$.

It remains to study the transitions between the consecutive terms in the expression (4-5) of $S_{3}^{d}$, that is, to compare the last entry in each term with the first entry in the next term. Four cases are to be considered, namely the special cases of the first two terms and of the final two terms, and the generic cases of the transitions from $\theta_{3, q} S_{2}^{d}$ to $S_{3}^{d, p+1}$ and from $S_{3}^{d, p}$ to $\theta_{3, q} S_{2}^{d}$.

As for the first two terms $\theta_{3,0} S_{2}^{d}=S_{2}^{d}$ and $\theta_{3,1} S_{2}^{d}=\sigma_{2} S_{2}^{d}$, the last entry in $S_{2}^{d}$ is $\sigma_{1}^{d}$, while the first entry in $\sigma_{2} S_{2}^{d}$ is $\sigma_{2}$, so $\sigma_{1}^{-d} \sigma_{2}$ is a quotient. For the last two terms $\theta_{3,2 d-1} S_{2}^{d}$ and $\theta_{3,2 d} S_{2}^{d}$, the last entry in $\theta_{3,2 d-1} S_{2}^{d}$ is $\theta_{3,2 d-1} \sigma_{1}^{d}$, while the first entry in $\theta_{3,2 d} S_{2}^{d}$ is $\theta_{3,2 d}$. Now, by (4-1), we have $\theta_{3,2 d-1} \sigma_{1}^{d} \sigma_{2}=\theta_{3,2 d} \sigma_{1}^{d}$, so $\sigma_{2} \sigma_{1}^{-d}$ expresses the quotient. 
Consider now the transition from $\theta_{3, q} S_{2}^{d}$ to $S_{3}^{d, q+1}$. The last entry in $\theta_{3, q} S_{2}^{d}$ is $\theta_{3, q} \sigma_{1}^{d}$, while, by Lemma 4.11, the first entry in $S_{3}^{d, q+1}$ is $\theta_{3, q} \sigma_{2}$. Hence $\sigma_{1}^{-d} \sigma_{2}$ represents the quotient. Finally, consider the transition from $S_{3}^{d, p}$ to $\theta_{3, q} S_{2}^{d}$. By Lemma 4.11 again, the last entry $x$ in $\theta_{3, q} S_{2}^{d}$ satisfies $x \sigma_{2}=\theta_{3, q} \sigma_{1}^{d}$, while the first entry in $\theta_{3, q} S_{2}^{d}$ is $\theta_{3, q}$. Hence $\sigma_{2} \sigma_{1}^{-d}$ represents the quotient.

Corollary 4.15. For each $d$ the sequence $S_{3}^{d}$ is <-increasing; so, in particular, it consists of pairwise distinct braids.

Proof. By definition, every word in $\boldsymbol{w}_{3}^{d}$ is $\sigma$-positive. Hence, by Property A, it does not represent 1.

As $S_{3}^{d}$ consists of pairwise distinct divisors of $\Delta_{3}^{d}$, Lemma 4.10 implies that every divisor of $\Delta_{3}^{d}$ occurs exactly once in $S_{3}^{d}$. Then, as $S_{3}^{d}$ is <-increasing, it must be the increasing enumeration of $\operatorname{Div}\left(\Delta_{3}^{d}\right)$, and the proof of Proposition 4.7 is complete.

Remark 4.16. Once we know that $S_{3}^{d}$ is the increasing enumeration of $\operatorname{Div}\left(\Delta_{3}^{d}\right)$ and that $\boldsymbol{w}_{3}^{d}$ is a $\sigma$-positive quotient sequence for $S_{3}^{d}$, we can count the 2-jumps in $S_{3}^{d}$ and obtain the value of $h_{2}\left(\Delta_{3}^{d}\right)$ directly. This amounts to forgetting about all $\sigma_{1}^{ \pm 1}$ in the construction of $\boldsymbol{w}_{3}^{d}$, and it is then fairly obvious that there only remains $2^{d}-2$ times $\sigma_{2}$.

4D. Larger values of $\boldsymbol{n}$. The same construction can be developed for $n=4$ and beyond. The general scheme is to define $S_{4}^{d}$ using an inductive rule

$$
S_{4}^{d}=\theta_{4,0} S_{3}^{d}+S_{4}^{d, 1}+\theta_{4,1} S_{3}^{d}+\cdots+\theta_{4,3 d-1} S_{3}^{d}+S_{4}^{d, 3 d}+\theta_{4,3 d} S_{3}^{d},
$$

where the intermediate factor $S_{4}^{d, p}$ is constructed by concatenating and translating convenient fragments of $S_{4}^{d-1}$. Owing to the inductive rule (3-6) satisfied by the number of elements $h_{1}\left(\Delta_{4}^{d}\right)$ of $\operatorname{Div}\left(\Delta_{4}^{d}\right)$, we can expect the generic entry of $S_{4}^{d-1}$ to be repeated six times in $S_{4}^{d}$, but with some entries from $S_{4}^{d-2}$ repeated three times only. After completing the inductive definition of $S_{4}^{d}$, showing that the sequence is <-increasing and counting its entries should be easy. As we have no complete description so far, we leave the question open here.

4E. A new construction for the linear ordering of $B_{3}$. In pursuing the approach described above, we were interested in connecting the Garside structure of $B_{n}$ with its linear ordering. In the process, we found something more: a new, independent construction of the braid ordering, at least for $B_{3}$, which is currently the only completed case.

As recalled in the introduction, the existence of the linear ordering of braids relies on two properties of braids, namely Property A and Property C. These properties have received a number of independent proofs [Dehornoy et al. 2002]. In 
particular, Property A has now a very short proof based on Dynnikov's coordinization for singular triangulations of a punctured disk [Dehornoy et al. 2002, Chapter 9]. As for Property C, no really simple proof exists so far. Even without the initial argument involving self-distributive algebra, the remaining arguments-the combinatorial proofs based on handle reduction or on Burckel's uniform tree approach, or the geometric proofs based on standardization of curve diagrams-all require some care. For now, it seems that the optimal proof of Property $\mathrm{C}$ is forthcoming.

Here is a direct application of our construction of the sequence $S_{3}^{d}$ :

Proposition 4.17. Property $C$ holds for $B_{3}$; that is, every nontrivial 3-braid admits a $\sigma$-positive or a $\sigma$-negative expression.

New proof. We take as an hypothesis that Property A is true, so that the relation $<$ is a partial ordering, but we do not assume that $<$ is linear. As every braid in $B_{3}$ is the quotient of two positive braids in $B_{3}^{+}$, proving Property $\mathrm{C}$ for $B_{3}$ amounts to proving that, if $x, y$ are arbitrary elements of $B_{3}^{+}$, then the quotient $x^{-1} y$ admits a $\sigma$-positive or a $\sigma$-negative expression.

Now the construction of $S_{3}^{d}$ is self-contained, as is that of $\boldsymbol{w}_{3}^{d}$. Then, by construction, every word in $\boldsymbol{w}_{3}^{d}$ is $\sigma$-positive. As any concatenation of $\sigma$-positive words is $\sigma$-positive, it follows that, if $x, y$ are any braids occurring in $\bigcup_{d} S_{3}^{d}$, then the quotient $x^{-1} y$ admits a $\sigma$-positive or a $\sigma$-negative expression, according to whether $x$ occurs before or after $y$ in $S_{3}^{d}$. To conclude Property $\mathrm{C}$ is true, it remains to check that each positive 3-braid occurs in $\bigcup_{d} S_{3}^{d}$. Because every entry of $S_{3}^{d}$ belongs to $\operatorname{Div}\left(\Delta_{3}^{d}\right)$, this is equivalent to proving that each divisor of $\Delta_{3}^{d}$ occurs in $S_{3}^{d}$. Property A guarantees that the entries of $S_{3}^{d}$ are pairwise distinct (Corollary 4.15), so it suffices to compare the length of $S_{3}^{d}$ with the cardinality of $\operatorname{Div}\left(\Delta_{3}^{d}\right)$, and this is what we made in Lemma 4.10.

The construction of $S_{3}^{d}$ gives more. The approach developed by S. Burckel [1997] introduces a convenient notion of normal braid words such that every positive braid admits exactly one normal expression. For 3-strand braids, the definition is as follows. Every positive 3-strand braid word $w$ can be written as an alternating product of blocks $\sigma_{1}^{e}$ and $\sigma_{2}^{e}$. Then we define the code of $w$ to be the sequence of the sizes of these blocks. To avoid ambiguity, we consider the last block to be a block of $\sigma_{1}$ 's, that is, we decide that the code of $\sigma_{1}$ is (1), while the code of $\sigma_{2}$ is $(1,0)$. For instance, the code of $\sigma_{2}^{2} \sigma_{1}^{3} \sigma_{2}^{5}$ is $(2,3,5,0)$.

Definition 4.18. A positive 3 strand braid word $w$ is said to be normal in the sense of Burckel if its code has the form $\left(e_{1}, \ldots, e_{\ell}\right)$ with $e_{k} \geqslant 2$ for $2 \leqslant k \leqslant \ell-2$.

Burckel [1997] shows that every positive 3-braid admits a unique normal expression and, moreover, that $x<y$ holds if and only if the normal form of $x$ is ShortLex-smaller than the normal form of $y$, where ShortLex refers to the 
variant of the lexicographic ordering of sequences in which the length is given priority: $\left(e_{1}, \ldots, e_{\ell}\right)<$ ShortLex $\left(e_{1}^{\prime}, \ldots, e_{\ell^{\prime}}^{\prime}\right)$ always holds for $\ell<\ell^{\prime}$ and, when $\ell=\ell^{\prime}$, it holds when $\left(e_{1}, \ldots, e_{\ell}\right)$ is lexicographically smaller than $\left(e_{1}^{\prime}, \ldots, e_{\ell^{\prime}}^{\prime}\right)$. Burckel's method defines an iterative reduction process on nonnormal braid words. Our current approach provides for a simpler method. First, a direct inspection shows:

Lemma 4.19. Let $\underline{S}_{3}^{d}$ be the sequence of braid words defined by the inductive rule (4-5). Then $\underline{S}_{3}^{d}$ consists of words that are normal in the sense of Burckel.

Then, by construction, every braid in $S_{3}^{d}$ is represented by a word of $\underline{S}_{3}^{d}$. As every positive 3-braid occurs in $\bigcup S_{3}^{d}$, we immediately deduce:

Proposition 4.20. Every positive 3-braid admits an expression that is normal in the sense of Burckel.

This in turn enables us to obtain a simple proof for the following deep, and so far not very well understood, result due to Laver [1996] and to Burckel [1997] for the ordinal type:

Corollary 4.21. The restriction of $<$ to $B_{3}^{+}$is a well-ordering of ordinal type $\omega^{\omega}$.

Proof. The ShortLex ordering of sequences of nonnegative integers is a wellordering of ordinal type $\omega^{\omega}$, so its restriction to codes of normal words in the sense of Burckel is a well-ordering as well. The type of the latter cannot be less than $\omega^{\omega}$, as one can easily exhibit an increasing sequence of length $\omega^{\omega}$.

Burckel's approach extends to all braid monoids $B_{n}^{+}$. Burckel introduces a convenient notion of a normal word, but the associated reduction process is very intricate. Hopefully, the above approach will provide a much simpler approach to completing the construction of the sequences $S_{4}^{d}$ and, more generally, $S_{n}^{d}$. In particular, once the correct definition is given, all subsequent proofs should reduce to easy inductions.

\section{Acknowledgement}

The author thanks the PJM production editors, Matt Cargo and Silvio Levy, for their suggestions.

\section{References}

[Birman 1974] J. S. Birman, Braids, links, and mapping class groups, Princeton University Press, Princeton, N.J., 1974. Annals of Mathematics Studies, No. 82. MR 51 \#11477

[Burckel 1997] S. Burckel, "The wellordering on positive braids", J. Pure Appl. Algebra 120:1 (1997), 1-17. MR 98h:20062 Zbl 0958.20032

[Burckel 1999] S. Burckel, "Computation of the ordinal of braids", Order 16:3 (1999), 291-304. MR 2001i:20075 Zbl 0980.20026 
[Burckel 2001] S. Burckel, "Syntactical methods for braids of three strands", J. Symbolic Comput. 31:5 (2001), 557-564. MR 2002b:20051 Zbl 0990.20022

[Carlitz et al. 1976] L. Carlitz, R. Scoville, and T. Vaughan, "Enumeration of pairs of permutations", Discrete Math. 14:3 (1976), 215-239. MR 53 \#156 Zbl 0322.05008

[Charney 1995] R. Charney, "Geodesic automation and growth functions for Artin groups of finite type”, Math. Ann. 301:2 (1995), 307-324. MR 95k:20055 Zbl 0813.20042

[Dehornoy 1994] P. Dehornoy, "Braid groups and left distributive operations", Trans. Amer. Math. Soc. 345:1 (1994), 115-150. MR 95a:08003 Zbl 0837.20048

[Dehornoy 1997] P. Dehornoy, "A fast method for comparing braids", Adv. Math. 125:2 (1997), 200-235. MR 98b:20060 Zbl 0882.20021

[Dehornoy 1999] P. Dehornoy, "Strange questions about braids", J. Knot Theory Ramifications 8:5 (1999), 589-620. MR 2000d:20052 Zbl 0933.20024

[Dehornoy 2002] P. Dehornoy, "Groupes de Garside", Ann. Sci. École Norm. Sup. (4) 35:2 (2002), 267-306. MR 2003f:20068 Zbl 1017.20031

[Dehornoy 2007] P. Dehornoy, "Combinatorics of normal sequences of braids", J. Combin. Theory Ser. A 114:3 (2007), 389-409. MR MR2310741 Zbl 1116.05006

[Dehornoy et al. 2002] P. Dehornoy, I. Dynnikov, D. Rolfsen, and B. Wiest, Why are braids orderable?, Panoramas et Synthèses 14, Société Mathématique de France, Paris, 2002. MR 2004e:20062 Zbl 1048.20021

[El-Rifai and Morton 1994] E. A. El-Rifai and H. R. Morton, "Algorithms for positive braids", Quart. J. Math. Oxford Ser. (2) 45:180 (1994), 479-497. MR 96b:20052 Zbl 0839.20051

[Epstein et al. 1992] D. B. A. Epstein, J. W. Cannon, D. F. Holt, S. V. F. Levy, M. S. Paterson, and W. P. Thurston, Word processing in groups, Jones and Bartlett, Boston, 1992. MR 93i:20036 Zbl 0764.20017

[Garside 1969] F. A. Garside, “The braid group and other groups”, Quart. J. Math. Oxford Ser. (2) 20 (1969), 235-254. MR 40 \#2051 Zbl 0194.03303

[Larue 1994] D. Larue, Left-distributive and left-distributive idempotent algebras, Ph.D. thesis, University of Colorado, Boulder, 1994.

[Laver 1996] R. Laver, "Braid group actions on left distributive structures, and well orderings in the braid groups", J. Pure Appl. Algebra 108:1 (1996), 81-98. MR 97e:20061 Zbl 0859.20029

[McCammond 2005] J. McCammond, “An introduction to Garside structures", Preprint, 2005, Available at http://www.math.ucsb.edu/ mccammon/papers/intro-garside.pdf.

[Solomon 1976] L. Solomon, "A Mackey formula in the group ring of a Coxeter group", J. Algebra 41:2 (1976), 255-264. MR 56 \#3104 Zbl 0355.20007

Received April 6, 2006.

\section{PATRICK DEHORNOY}

LAboratoire de Mathématiques Nicolas Oresme UMR 6139

UNIVERSITÉ DE CAEN

14032 CAEN

FRANCE

dehornoy@math.unicaen.fr

www.math.unicaen.fr/ dehornoy 